\title{
Observable Spectra of Induced Gravitational Waves from Inflation
}

\author{
Laila Alabidi $^{a}$ Kazunori Kohri $^{b, c}$ Misao Sasaki $^{a}$ Yuuiti Sendouda $^{d}$ \\ ${ }^{a}$ Yukawa Institute for Theoretical Physics, Kyoto University, Kyoto 606-8502, Japan \\ ${ }^{b}$ Cosmophysics Group, Theory Center, IPNS, KEK, Tsukuba 305-0801, Japan \\ ${ }^{c}$ The Graduate University for Advanced Study (Sokendai), Tsukuba 305-0801, Japan \\ ${ }^{d}$ Graduate School of Science and Technology, Hirosaki University, Hirosaki, Aomori 036- \\ 8561, Japan \\ E-mail: laila@yukawa.kyoto-u.ac.jp, kohri@post.kek.jp, \\ misao@yukawa.kyoto-u.ac.jp, sendouda@cc.hirosaki-u.ac.jp
}

\begin{abstract}
Measuring the primordial power spectrum on small scales is a powerful tool in inflation model building, yet constraints from Cosmic Microwave Background measurements alone are insufficient to place bounds stringent enough to be appreciably effective. For the very small scale spectrum, those which subtend angles of less than 0.3 degrees on the sky, an upper bound can be extracted from the astrophysical constraints on the possible production of primordial black holes in the early universe. A recently discovered observational by-product of an enhanced power spectrum on small scales, induced gravitational waves, have been shown to be within the range of proposed space based gravitational wave detectors; such as NASA's LISA and BBO detectors, and the Japanese DECIGO detector. In this paper we explore the impact such a detection would have on models of inflation known to lead to an enhanced power spectrum on small scales, namely the Hilltop-type and running mass models. We find that the Hilltop-type model can produce observable induced gravitational waves within the range of $\mathrm{BBO}$ and DECIGO for integral and fractional powers of the potential within a reasonable number of $e$-folds. We also find that the running mass model can produce a spectrum within the range of these detectors, but require that inflation terminates after an unreasonably small number of $e$-folds. Finally, we argue that if the thermal history of the Universe were to accomodate such a small number of $e$-folds the Running Mass Model can produce Primordial Black Holes within a mass range compatible with Dark Matter, i.e. within a mass range $10^{20} \mathrm{~g} \lesssim M_{\mathrm{BH}} \lesssim 10^{27} \mathrm{~g}$.
\end{abstract}

Keywords: Inflation, Primordial Black Holes, Induced Gravitational Waves, DECIGO, BBO, LISA 


\section{Contents}

1 Introduction 1

2 Induced Gravitational Waves $\quad 2$

3 Primordial Black Holes $\quad 3$

$\begin{array}{lll}4 & \text { Inflationary Parameters } & 7\end{array}$

5 The Models of Inflation $\quad 8$

5.1 Hilltop-type model 8

5.2 The Running Mass Model 10

6 Results and Discussion 12

$\begin{array}{llr}7 & \text { Conclusions } & 18\end{array}$

$\begin{array}{lr}\text { A Induced Gravitational Waves } & 18\end{array}$

A.1 The $\tau$ integrals $\quad 22$

A.2 The energy density of second order gravitational waves 23

B The coefficients of the $\tau$ integral $\quad 24$

C The coefficients of the Hilltop Model 25

$\begin{array}{lr}\text { D The sensitivity curves } & 26\end{array}$

\section{Introduction}

One of the main goals of cosmology is to uncover a unique model of inflation, an important achievement that will lead to a fuller understanding of the dynamics of the very early universe and allow us to better compare with fundamental theory. To fully probe the inflationary potential, an accurate measure of the primordial spectrum on all scales is required. To date, Cosmic Microwave Background (CMB) experiments such as COBE and WMAP [1], Baryon Acoustic Oscillations (BAO) in Galaxy Survey experiments (such as SDSS [2]) and Super Novae (SN) [3] observations have constrained the spectrum on large scales to $\mathcal{P}_{\zeta}=$ $2.325 \times 10^{-9}$ with an uncertainty of $\pm 0.098 \times 10^{-9}$. Here, by large scale we mean scales which subtend angles on the sky of more than $\theta=0.3^{\circ}$. Bounds on the smaller scale spectrum arise from such sources as the Lyman- $\alpha$ forest which measures this spectrum via a trace of the Baryonic power spectrum from the intergalactic medium (e.g. Ref. [4]) ${ }^{1}$, weak lensing (e.g. Ref. [6]), the Sunyaev-Zeldovich effect (e.g. Ref. [7]), bounds from Ultra compact Mini Halos [8-12] and the astrophysical bounds on the production of Primordial Black Holes (PBHs) $[13,14]$.

The $\mathrm{PBH}$ bound constrains the spectrum on the smallest scales, those which exit the horizon towards the end of inflation, and it is the uncertainty in this bound which allows for

\footnotetext{
${ }^{1}$ See Ref. [5] for the latest analysis of the Lyman- $\alpha$ forest
} 
some leeway in inflation model building. In previous work [15-18] it has been shown that Hilltop-type models and their ilk can give rise to an enhanced spectrum towards the end of inflation, within the observational bounds of PBH production. An actual measurement of this feature would prove decisive in inflation model discrimination ${ }^{2}$. References $[20-$ 26] have shown that the primordial scalar spectrum can result in the production of what are known as induced gravitational waves ${ }^{3}$. These gravitational waves have an enhanced energy density for an enhanced scalar spectrum which can exceed that of the primordial tensor spectrum for small field models of inflation. This opens up another avenue for fully probing the primordial spectrum and placing constraints on the cosmological and inflationary parameters (e.g. Ref [28]).

In this scenario, scalar perturbations re-enter the horizon during Big Bang Nucleosynthesis perturbing the background metric and inducing a tensor perturbation. The modes which enter the horizon during radiation domination generate gravitational waves on scales accessible to space based gravitational wave detectors [26, 28] such as LISA [29], BBO and DECIGO [30, 31]. The scalar perturbations which enter the horizon during matter domination generate an induced gravitational wave spectrum accessible to CMB experiments [32], and since these waves do not redshift, their spectrum is scale dependent [26], making them distinguishable from their primordial, scale independent, brethren. We are interested in the former scales, which leave the horizon towards the end of inflation, and re-enter during the radiation era.

Previous work [33-35] calculated the induced gravitational wave spectra for step and power law primordial spectra, and reference [36] calculated the induced GW-spectrum of the running mass model. In this work we calculate the spectra of induced gravitational waves for various parameter choices in both the running mass and Hilltop-type models. We find that in fact, the Hilltop-type model can result in a significant amplitude of gravitational waves, measurable by the BBO/DECIGO and cross-correlated DECIGO detectors.

In Section 2 we briefly present the equations for Induced Gravitational Waves, then in Section 3 we evaluate the upper bounds on the scalar spectrum from the possible production of Primordial Black Holes. In Section 4 we summarise the inflationary parameters and their observational bounds. In Section 5 we present the models of inflation that we are analysing and their parameters. In Section 6 we present our results with some discussion. Finally, in Section 7 we summarise the main results of this work.

The following conventions are utilised in this paper: $\tau$ refers to conformal time and is related to proper time $t$ as $d \tau=d t / a, a$ is the scale factor, and the conformal Hubble parameter $\mathcal{H}$ is related to the Hubble parameter $H \equiv \dot{a} / a$ as $\mathcal{H}=a H$. Scales are denoted by $k$, are given in units of inverse megaparsec $\mathrm{Mpc}^{-1}$ and are related to physical frequency $f$ as $f=c k /(2 a \pi)$ where $c$ is the speed of light. We assume a radiation dominated universe at the time of the formation of the gravitational waves, in which case we have $a=a_{0}\left(\tau / \tau_{0}\right)$, $\mathcal{H}=\tau^{-1}$, and the scale at re-entry is $k=\tau^{-1}$.

\section{Induced Gravitational Waves}

After Inflation has ended, scalar perturbations begin the re-enter the horizon and interact in such a a way as to induce gravitational waves. These gravitational waves have a spectrum

\footnotetext{
${ }^{2}$ For example, Ref. [19] uses the $\mathrm{PBH}$ bound to constrain the cosmological observables

${ }^{3}$ See also Ref. [27] for an alternative scenario, where gravitational waves are induced by particle production during inflation
} 
that is dependent on scale and is given as

$$
P_{h}(k)=\frac{1}{a^{2}} \int_{0}^{\infty} d \tilde{k} \int_{-1}^{1} d \mu \frac{k^{3} \tilde{k}^{3}}{|\mathbf{k}-\tilde{\mathbf{k}}|^{3}}\left(1-\mu^{2}\right)^{2} P(|\mathbf{k}-\tilde{\mathbf{k}}|) P(\tilde{k}) I_{1}(k, \tilde{k}, \tau) I_{2}(k, \tilde{k}, \tau)
$$

derived in appendix (A). Where $P(k)$ is the scalar spectrum given by the model of inflation, $\mu$ is the cosine of the angle between the two modes and $I_{1}$ and $I_{2}$ are the time integrals given in appendix (A). In this paper we work with the parameters $v=\tilde{k} / k, y=\sqrt{1+v^{2}-2 v \mu}$ and $x=k \tau$, in which case the spectrum of induced gravitational waves takes the form

$$
P_{h}(k)=\frac{k^{2}}{x^{2}} \int_{0}^{\infty} d v \int_{|v-1|}^{|v+1|} d y \frac{v^{2}}{y^{2}}\left(1-\mu^{2}\right)^{2} P(k v) P(k y) \tilde{I}_{1} \tilde{I}_{2} .
$$

What is important to note here is that the time integrals, given in Eq. (A.35) are independent of the model of inflation, and depend only on the epcoh of evaluation. In this paper we work only with gravitational waves induced during the radiation era, and therefore the time integrals are given by Eqs. (A.36) and (A.37). Their behaviour is plotted in Fig. (11), and as is clear, the envelope of the integrals is constant for a fixed scale and rapidly decay to zero for $\tilde{k} \gg k$.

Since gravitational wave detectors will measure the amplitude of the energy density of gravitational waves, we will be presenting our results in terms of the dimensionless variable $\Omega_{G W}$ which defines the variation of the energy density with respect to the logarithm of the scale. We discuss this parameter more in appendix (A.2), for now we present the form of $\Omega_{G W}$ for scales which re-enter the horizon during the radiation era

$$
\Omega_{G W}=\frac{1}{1+z_{e q}} P_{h}(k)
$$

where $e q$ denotes matter-radiation equality.

\section{Primordial Black Holes}

In this section we evaluate upper bounds on $\mathcal{P}_{\zeta}$ from $\mathrm{PBH}$ formation, which is severely constrained by various astrophysical and cosmological observations [13].

In the simplest model, PBHs are formed due to instantaneous gravitational collapse during the radiation-dominated era. Their mass is given in terms of the energy within the sound horizon at the time of formation, $M \equiv c^{3} /(2 G H(t))$, as $M_{\mathrm{BH}}=\gamma M$, where $\gamma$ is a numerical factor to represent uncertainty. The corresponding comoving wave number of a density perturbation is

$$
k_{\mathrm{BH}}=a H \simeq \frac{k_{\mathrm{eq}}}{2^{1 / 4}}\left(\frac{g_{*}}{g_{* \mathrm{eq}}}\right)^{-1 / 12}\left(\frac{M}{M_{\mathrm{eq}}}\right)^{-1 / 2} \quad\left(M \ll M_{\mathrm{eq}}\right),
$$

where

$$
k_{\mathrm{eq}} \equiv a_{\mathrm{eq}} H_{\mathrm{eq}} \approx 0.00974 \mathrm{Mpc}^{-1}, \quad M_{\mathrm{eq}} \equiv \frac{c^{3}}{2 G H_{\mathrm{eq}}} \approx 6.67 \times 10^{50} \mathrm{~g} .
$$


For a Gaussian-distributed density perturbation ${ }^{4}$, the energy fraction going into collapsed objects, $\beta(M)$, is related to the mass variance at horizon entry, $\sigma(M)^{2}$ via the formula

$$
\beta(M)=\gamma \frac{2}{\sqrt{2 \pi} \sigma(M)} \int_{1 / 3}^{1} \mathrm{~d} \delta \exp \left(-\frac{\delta^{2}}{2 \sigma(M)^{2}}\right) \approx \gamma \operatorname{erfc}\left(\frac{1 / 3}{\sqrt{2} \sigma(M)}\right),
$$

where the prefactor 2 embodies the Press-Schechter prescription. Equation (3.3) is numerically inverted to give the value of $\sigma$ for a given upper limit of $\beta$.

The mass variance evaluated at horizon entry (when $a H=k_{\mathrm{BH}}$ holds) is

$$
\sigma(M)^{2}=\frac{16}{81} \int_{0}^{\infty} W\left(k / k_{\mathrm{BH}}\right)^{2} T(k)^{2}\left(\frac{k}{k_{\mathrm{BH}}}\right)^{4} \mathcal{P}(k) \frac{\mathrm{d} k}{k},
$$

where $W$ is the window function, $T$ is the transfer function and $\mathcal{P}$ is the power spectrum of the curvature perturbation in the comoving gauge. We choose a Gaussian window function $W(x)=\mathrm{e}^{-x^{2} / 2}$ and assume $T(k)=1$ for simplicity. Since the window function has a sharp cutoff, we only need to evaluate the power spectrum around $k_{\mathrm{BH}}$ to calculate the mass variance on the relevant scale. Incidentally, the comoving curvature perturbation is identical to the curvature perturbation in the uniform-density gauge $\zeta$ (up to a sign difference) on superhorizon scales. Thus $\mathcal{P}$ in the above equation can simply be replaced by the spectrum of $\zeta$, denoted by $\mathcal{P}_{\zeta}$. If the spectrum is locally scale invariant, i.e. $n(k) \approx 1$ in the neighbourhood of $k_{\mathrm{BH}}$, as is generally expected in various inflationary models, then the mass variance is estimated as

$$
\sigma(M)^{2} \approx \frac{8}{81} \mathcal{P}_{\zeta}\left(k_{\mathrm{BH}}\right)
$$

Using this equation and Eq. (3.3), we can estimate the upper limit of $\mathcal{P}_{\zeta}$ from that on the fraction $\beta(M)=\beta\left(M_{\mathrm{BH}} / \gamma\right)$; which is given in Fig. 9 of Ref. [13]. In Fig. (1), we plot the obtained upper limit on $\mathcal{P}_{\zeta}$ as a function of $k$.

\footnotetext{
${ }^{4}$ Refer to Refs. [37-42] for the alternative scenario where a non-gaussian distributed density perturbation is considered.
} 
Table 1. Upper limits on PBH formation from various observations. Most of them come from non-detections (NDs) of astrophysical phenomena or effects which would be detectable if there were a sufficient number of PBHs. See [13] for details. Note that the structure on scales less than $10^{4}\left[\mathrm{Mpc}^{-1}\right]$ may be further constrained in the future by the upcoming PIXIE experiment [43].

\begin{tabular}{c|l}
\hline \hline $\begin{array}{c}\text { Wavenumber } \\
\gamma^{1 / 2} k\left[\mathrm{Mpc}^{-1}\right]\end{array}$ & Constraints \\
\hline$<3.9$ & Density of PBHs $\left(\Omega_{\mathrm{PBH}}\right)<0.25$ \\
$3.9 \times 10^{0}-1.5 \times 10^{4}$ & No excessive dynamical friction in the Galactic halo \\
$1.5 \times 10^{4}-3.0 \times 10^{4}$ & ND of Poisson fluctuations in Lyman- $\alpha$ forest \\
$3.0 \times 10^{4}-2.1 \times 10^{5}$ & ND of wide binary disruption in the Galaxy \\
$2.1 \times 10^{5}-6.1 \times 10^{5}$ & $\Omega_{\mathrm{PBH}}<0.25$ \\
$6.1 \times 10^{5}-4.7 \times 10^{6}$ & ND of microlensed quasars \\
$4.7 \times 10^{6}-4.7 \times 10^{9}$ & Lack of MACHO events \\
$4.7 \times 10^{9}-1.5 \times 10^{13}$ & $\Omega_{\mathrm{PBH}}<0.25$ \\
$1.5 \times 10^{13}-4.7 \times 10^{14}$ & ND of femto/picolensed gamma-ray bursts \\
$4.7 \times 10^{14}-7.5 \times 10^{14}$ & $\Omega_{\mathrm{PBH}}<0.25$ \\
$7.5 \times 10^{14}-7.5 \times 10^{15}$ & ND of extragalactic gamma-rays $(\mathrm{EGR})$ \\
$7.5 \times 10^{15}-9.2 \times 10^{15}$ & ND of Galactic gamma-rays \\
$9.2 \times 10^{15}-1.6 \times 10^{16}$ & ND of EGR \\
$1.6 \times 10^{16}-4.2 \times 10^{16}$ & No damping of small-scale CMB anisotropy \\
$4.2 \times 10^{16}-6.4 \times 10^{18}$ & Standard BBN \\
$6.4 \times 10^{18}-2.8 \times 10^{21}$ & Density of the lightest SUSY particle $($ LSP $)<0.25$ \\
& $($ assuming $100 \mathrm{GeV}$ for LSP's mass $)$ \\
$2.8 \times 10^{21}-2.1 \times 10^{23}$ & Density of Planck mass relics $<0.25$ \\
& (assuming $\mathcal{O}\left(10^{16}\right.$ GeV) for the reheating temperature) \\
\hline \hline
\end{tabular}




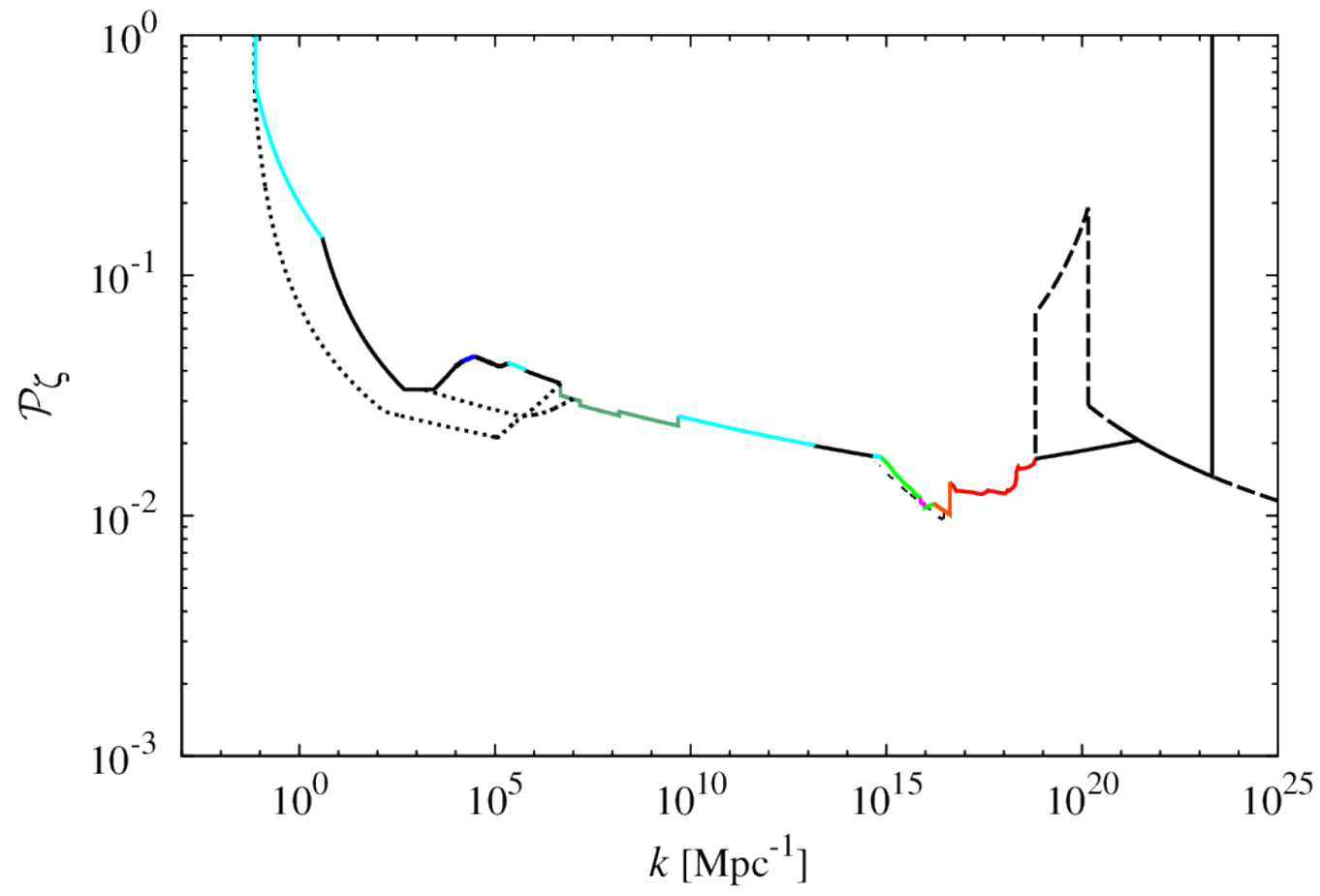

Figure 1. Upper limits on $\mathcal{P}_{\zeta}$ from PBH constraints. For simplicity, $\gamma$ is set to be unity. The different coloured solid lines correspond to bounds from different phenomena and experiments as summarised in table 1. For comparison, the dotted lines are included to indicate CMB constraints on accreting PBHs; the thin-dashed line illustrates a potential constraint from future $21 \mathrm{~cm}$ line experiments; the thick-dashed line is the limit necessary to avoid excessive generation of entropy and Planck mass relics $\left(k \lessgtr 2.1 \times 10^{23} \mathrm{Mpc}^{-1}\right)$. For the latter the reheating temperature is assumed to be higher than $10^{16} \mathrm{GeV}$. See [13] for details. 


\section{Inflationary Parameters}

We consider only single-field canonical models of inflation, where the accelerated expansion of the universe is driven by a flat potential, and a slowly changing scalar field (slowly rolling inflaton). To parametrize models of inflation, slow roll parameters [44] are used, defined as:

$$
\begin{aligned}
\epsilon & =\frac{m_{\mathrm{Pl}}^{2}}{2}\left(\frac{V_{, \varphi}}{V}\right)^{2} \\
\eta & =m_{\mathrm{Pl}}^{2} \frac{V_{, \varphi \varphi}}{V} \\
\xi^{2} & =m_{\mathrm{Pl}}^{4} \frac{V_{, \varphi} V_{, \varphi \varphi \varphi}}{V^{2}}
\end{aligned}
$$

where $V$ is the potential, and derivatives are with respect to the inflaton field $\varphi$. From this we can write down the observational parameters, the spectral index $n_{s}$, the running of the spectral index $n_{s}^{\prime}$ and the scalar spectrum $\mathcal{P}_{\zeta}$ :

$$
\begin{aligned}
n_{s} & =1+2 \eta-6 \epsilon \\
n_{s}^{\prime} & =16 \epsilon \eta-24 \epsilon^{2}-2 \xi^{2} \\
\mathcal{P}_{\zeta} & =\frac{1}{24 \pi^{2} m_{\mathrm{Pl}}^{4}} \frac{V}{\epsilon}
\end{aligned}
$$

where we neglect to mention the signature of primordial gravitational waves, the tensor to scalar ratio, since we are only considering small field models and as such this parameter is negligibly small.

The next ingredient required is the number of $e$-folds, the logarithmic ratio of the scale factor at two different times, in this case between the end of inflation and the time of horizon exit. This is related to the potential in the slow roll limit as:

$$
N \simeq m_{\mathrm{Pl}}^{-2} \int_{\varphi_{e}}^{\varphi_{*}} \frac{V}{V^{\prime}} d \varphi
$$

and to the corresponding horizon exiting scale as [44]:

$$
N\left(k_{0}\right)-N(k)=\ln \left(\frac{0.002}{k}\right) .
$$

where $k_{0}=0.002 \mathrm{Mpc}^{-1}$ is the pivot scale, and in this paper we effectively take $N\left(k_{0}\right)=0$.

We use the latest data release from the WMAP mission [1], for the WMAP data combined with $\mathrm{BAO}$ and $\mathrm{SN}$ data with a null tensor prior. This gives us the following bounds on the spectral index, and running of the spectral index at the $2 \sigma$ confidence limit:

$$
\begin{array}{r}
-0.093<1-n_{s}<0.076 \\
-0.061<n_{s}^{\prime}<0.017
\end{array}
$$

in this paper we take $n_{s}=0.95$ or $n_{s}=0.96$. 


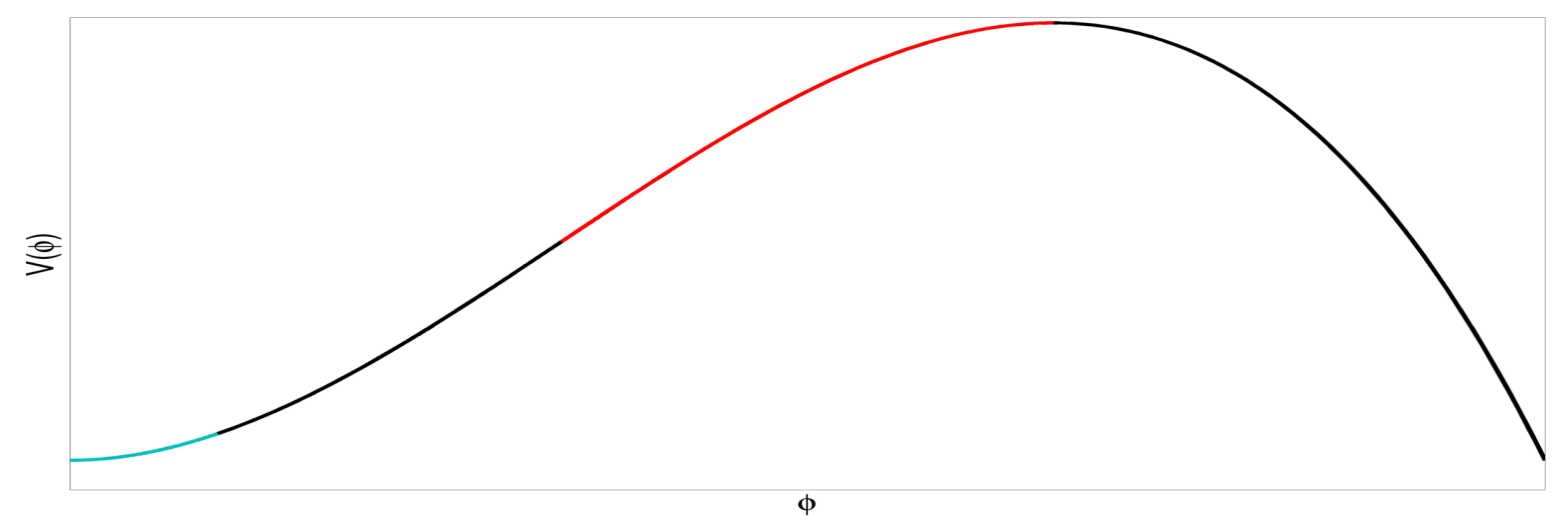

Figure 2. An illustration of the Hilltop-type and running models. In our scenario scales of cosmological interest during the hilltop regime (indicated with red), and the end of inflation occurs once the inflaton has reached a flatter region of the potential (indicated with blue).

\section{The Models of Inflation}

In this paper we analyse two models of inflation, the Hilltop-type model and the running mass model. Both models have a hilltop regime, an inflection point to one side of the hilltop and a steep slope to the other side. In the set-up of interest, scales of cosmological interest leave the horizon while the inflaton is on the inflection point side. The inflaton then proceeds to roll down the potential, past the inflection point and towards a region of further flatness, $\epsilon \rightarrow 0$, as illustrated in fig. 2. This means that our spectrum at the pivot scale will satisfy the WMAP bounds while still increasing on the smaller scales. Ref.[45] analyse various models of inflation and conclude that only the running mass model can allow for PBH formation, and we note that the hilltop model is phenomenologically equivalent to the running mass model. We begin by introducing the Hilltop-type model and the associated scalar spectrum and then move onto the running mass model and its scalar spectrum. We do not explicitly define a mechanism for the end of inflation or for the necessary subsequent reheating. We demand that inflation is ended abruptly after a specified number of $e$-folds, e.g. terminated by a waterfall field, and that reheating is instant.

\section{$5.1 \quad$ Hilltop-type model}

The phenomenological form of the potential [16] is given as:

$$
V=V_{0}\left(1+\eta_{p} \varphi^{p}-\eta_{q} \varphi^{q}\right)
$$

where $\eta_{p}$ and $\eta_{q}$ are referred to as the mass-coupling terms and self coupling powers $p$ and $q$ are required to satisfy $p<q$. This condition is to guarantee the hilltop form of the potential. Such a potential form also appears in supergravity models (e.g. ref. [16, 46-50]). The model has four degrees of freedom, with only weak constraints from fundamental theory. Therefore for each $\{p, q\}$ combination we scan the $\left\{\eta_{p}, \eta_{q}\right\}$ parameter space as follows

- We pre-set both $\eta_{p}$ and $\eta_{q}$ to be less than one.

- From the range of values $\{0,1\}$, the parameter range of $\left\{\eta_{p}, \eta_{q}\right\}$ is reduced by requiring that, at the pivot scale, the spectral index and the running of the spectral index are within the WMAP bounds, $n_{s}=0.95$ and $n_{s}^{\prime}<0.017$. 


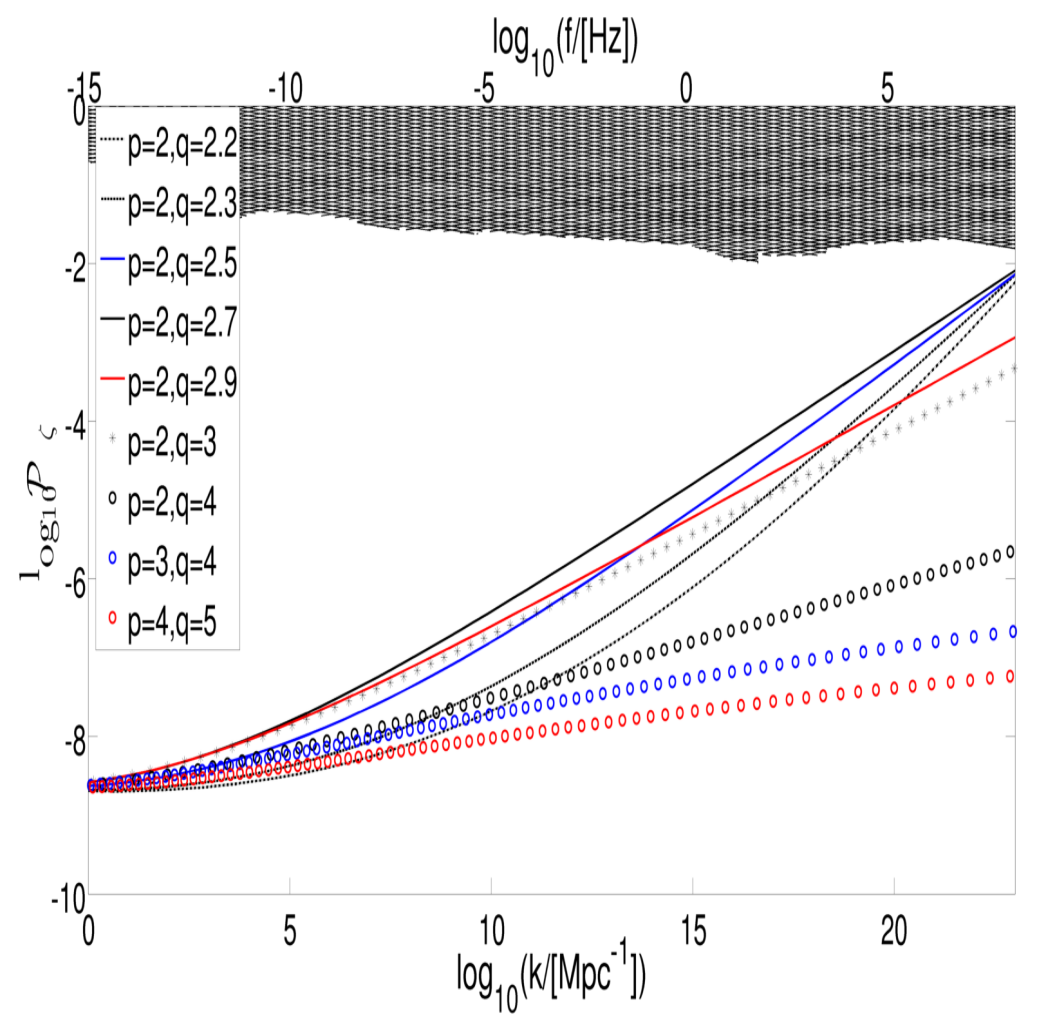

Figure 3. The scalar spectra of the hilltop model terminating at $N=65\left(\log _{10}\left(k /\left(0.002\left[\mathrm{Mpc}^{-1}\right]\right)\right) \sim\right.$ 28.2) while ensuring that $\mathcal{P}(N=60)$ is less than the $\mathrm{PBH}$ bound at that scale. The cross-hatched region is the $\mathrm{PBH}$ constraint.

- The parameter range is further reduced by rejecting mass-coupling combinations for which the field value at horizon exit is greater than the Planck scale; i.e. we demand $\phi_{*}<m_{\mathrm{Pl}}$.

- The range is then reduced to unique values of $\left\{\eta_{p}, \eta_{q}\right\}$ for each model by demanding that, after $\mathrm{N} e$-folds of inflation, the spectrum is close to but still less than the PBH bound at that $e$-fold, $\mathcal{P}(N) \lesssim \mathcal{P}_{\mathrm{PBH}}$. The exception to this is the $N=65$ case, for which we refer the reader to the following paragraph.

The fourth step essentially selects the unique mass-coupling values which maximize the spectrum at the end of inflation, compatible with the PBH bound. As we mentioned, the exception to the requirement in the fourth step is the case where inflation terminates at $N=65$. Since the PBH bound only applies up $N \sim 60\left(k \sim 10^{23}\right)$, we maximise the spectrum at the intermediate scale and then allow the model to continue to evolve until $N=65$. Clearly the spectra at $N=60$ for the $N=60$ and $N=65$ model will be the same, the difference is that for the $N=60$ model $\mathcal{P}_{\zeta}(N>60)=0$ while for the $N=65$ model $\mathcal{P}_{\zeta}(N>60) \neq 0$. A-priori we expect the final results for the GW spectrum to be the same in the range of interest, since the time integra asymptote to zero for $k>\tilde{k}$, but we evaluate the $N=65$ case anyway as a consistency check of our numerics. We tabulate the parameters and predictions for $n_{s}^{\prime}$ and $V_{0}$ in Appendix (C) and plot the results for the first order spectrum for $N=55, N=60$ and $N=65$ in Figures (5), (4) and (3). 


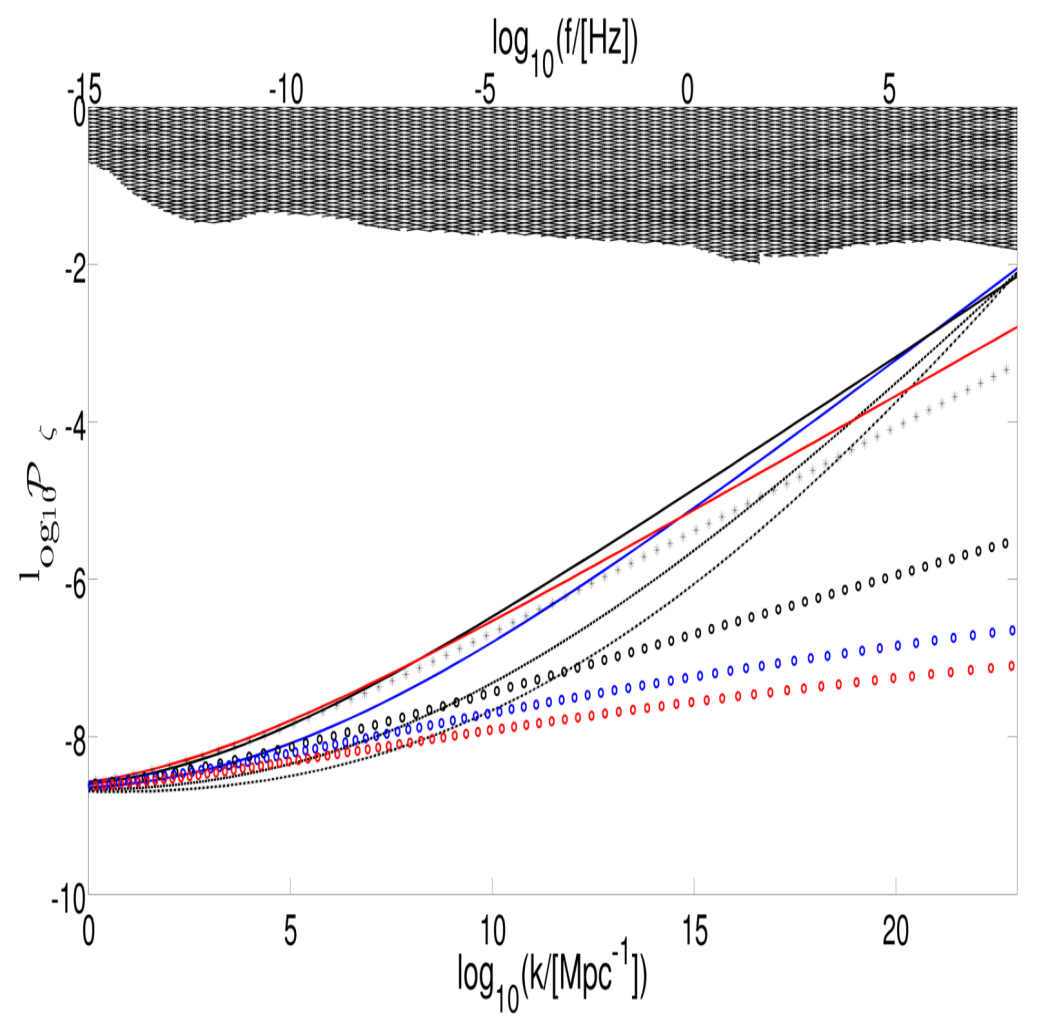

Figure 4. Scalar spectra from hilltop inflation with self-coupling powers labelled in the legend in Fig. (3). The plot corresponds to maximising the spectrum at $N=60\left(\log _{10}\left(k /\left(0.002\left[\mathrm{Mpc}^{-1}\right]\right)\right) \sim 26\right)$. The cross-hatched region is the PBH constraint.

\subsection{The Running Mass Model}

This model $[15,51-57]$ is a $\varphi^{2}$ model only with a mass term which varies with $\varphi$. The induced gravitational wave spectrum in this model was originally evaluated in Ref. [36]. The potential is of the form:

$$
\frac{V}{V_{0}}=1-\frac{B_{0}}{2} \varphi^{2}+\frac{A \varphi^{2}}{2(1+\alpha \ln (\varphi))^{2}} .
$$

We know from previous work [18] that the parameter values $A=2.4, B_{0}=2.42$, $\alpha=0.01$ satisfy WMAP bounds and lead to PBHs at the end of inflation. In this case, for $n_{s}=0.95$, then $V_{0}^{1 / 4}=0.003 m_{\mathrm{Pl}}$ and $n_{s}^{\prime}=0.0023$. We also consider the case of $n_{s}=0.96$ and $n_{s}^{\prime}=0.005$, which requires $A=3.1, B_{0}=3.08$ and $\alpha=0.01$ and has an inflationary energy scale of $V_{0}^{1 / 4}=0.004 m_{\mathrm{Pl}}$. The latter are the parameters analysed in Ref. [36] and serve as a tool of comparison between our work and theirs. We also evaluate the spectra for the range of parameters which maximise the spectrum in the range $k=\left\{10^{10}, 10^{14}\right\}\left[\mathrm{Mpc}^{-1}\right]$. This is because PBHs forming within this range are possible canditates for Dark Matter. The parameters maximising the spectrum at $k=10^{10}\left[\mathrm{Mpc}^{-1}\right]$ are $A=4.4, B=4.2, \alpha=0.01$, and they satisfy $n_{s}=0.96$ and $n_{s}^{\prime}=0.012$ with an inflationary energy scale of $V_{0}^{1 / 4}=0.0011 m_{\mathrm{Pl}}$. Maximising the spectrum at $k=10^{14}\left[\mathrm{Mpc}^{-1}\right]$ requires $A=3.4, B=0.01$ and $\alpha=0.01$, satisfying $n_{s}=0.96, n_{s}^{\prime}=0.0067$ with an inflationary energy scale of $V_{0}^{1 / 4}=0.0008 m_{\mathrm{Pl}}$. We also investigated whether this model could maximise the spectrum near $k=10^{5}\left[\mathrm{Mpc}^{-1}\right]$ since these PBHs would be candidates for seeds of SuperMassive Black Holes [58, 59], however we 


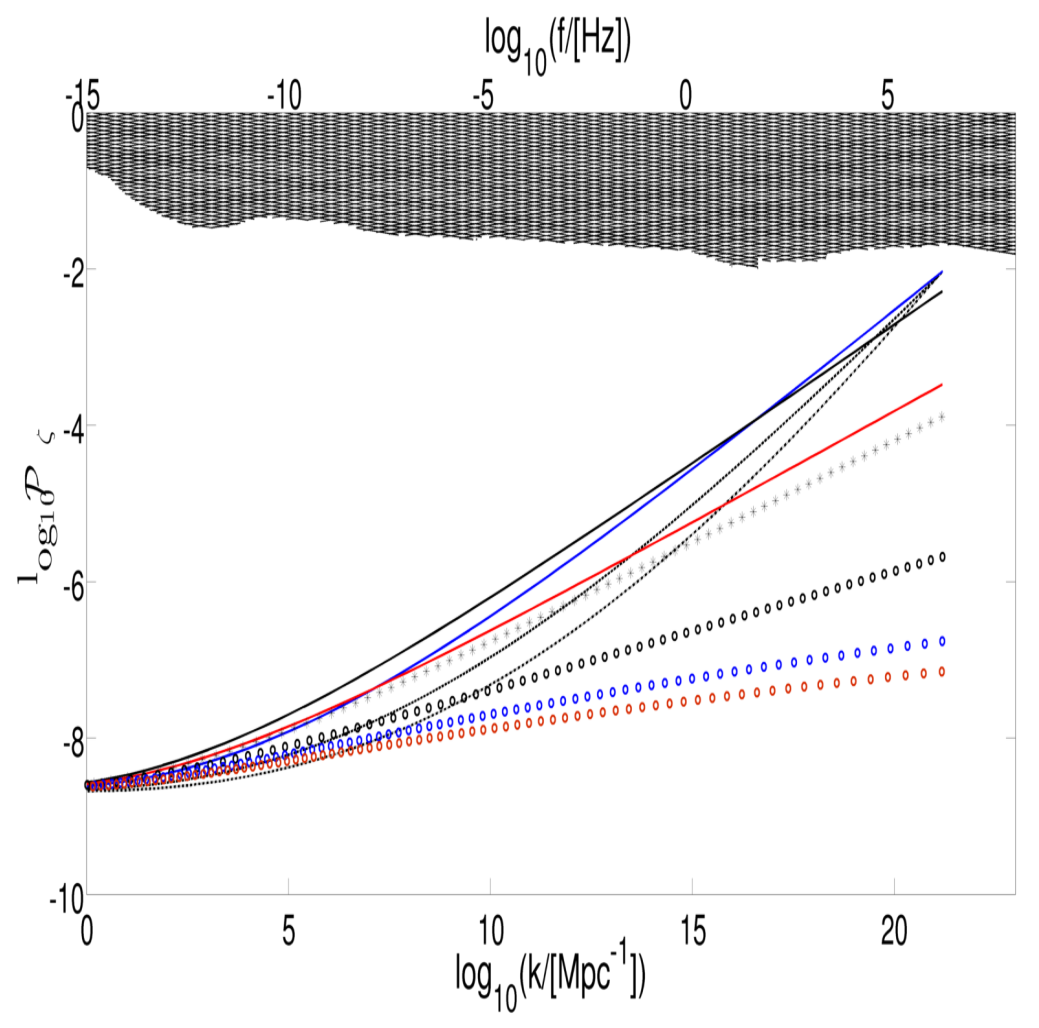

Figure 5. Scalar spectra from hilltop inflation with self-coupling powers labelled in the legend in Fig. (3). The plot corresponds to maximising the first order spectrum at $N=55$ $\left(\log _{10}\left(k /\left(0.002\left[\mathrm{Mpc}^{-1}\right]\right)\right) \sim 23.9\right)$, and the cross hatched region is the PBH bound

found that this would require a running greater than that allowed by WMAP. The primordial scalar spectra for these choices are plotted in Fig. (6). 


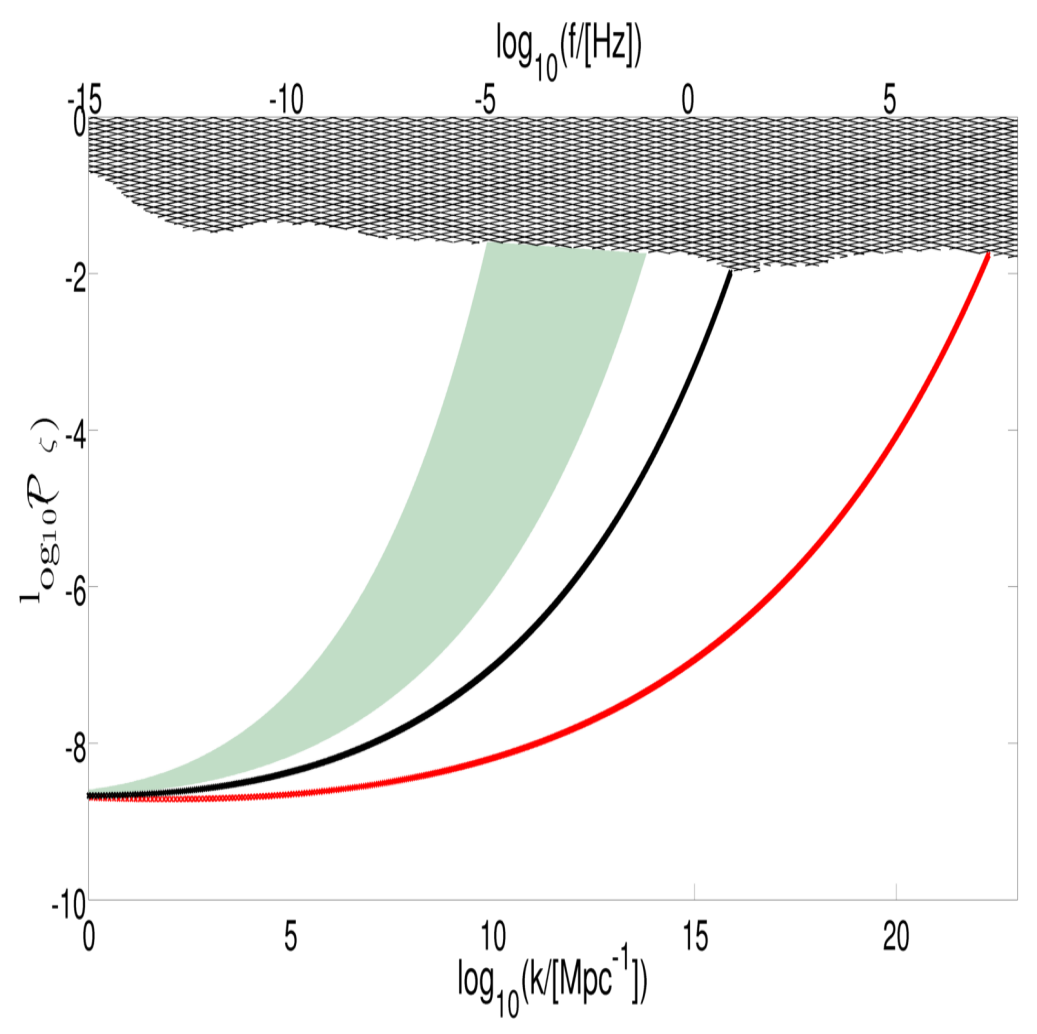

Figure 6. Spectra for the running mass models all satisfying $n_{s}=0.96$. The $n_{s}^{\prime}=0.005$ model (black crosses) requires a termination of inflation at $N \sim 43\left(\log _{10}\left(k /\left(0.002\left[\mathrm{Mpc}^{-1}\right]\right)\right) \sim 16\right)$ for compatibility with the PBH bound, and the $n_{s}^{\prime}=0.002$ model (red crosses) requires only a termination at $N \sim 64$. The hatched line is the $\mathrm{PBH}$ bound and both parameter combinations lead to the production of PBHs towards the end of inflation. The green shaded region corresponds to range of parameters in the running mass model which result in the production of PBHs whose energy density agrees with that of Dark Matter. The range corresponds to $10<\log _{10}\left(k /\left[\mathrm{Mpc}^{-1}\right]\right)<14$, where the lower $k$ value represents $n_{s}^{\prime}=0.012$, and inflation terminating at $N=29$ while the upper $k$ value represents $n_{s}^{\prime}=0.0067$, and inflation terminating at $N=38.5$.

\section{Results and Discussion}

In both cases we begin by computing the integral over $y$ in Eq. (2.2) for a range of $k=$ $10^{4} \cdots 10^{25} \mathrm{Mpc}^{-1}$. This is done using the known results of Eq. (A.35) for a radiation era, Eqs. (A.36) and (A.37), and the Second Euler- Maclaurin summation formula [60]. This method allows us to avoid integrating over a singularity which occurs when $v=1$ by aiding us in 'avoiding' the endpoints. We introduce a sudden cutoff approximation for the calculation of the induced gravitational wave spectrum, effectively we assume that structure on very small scales $k>k_{\text {end }}$ is non-existent, $\mathcal{P}_{\zeta}\left(k>k_{\text {end }}\right) \sim 0$. To ensure that the $\mathrm{PBH}$ bound is not violated, we perform a comparative analysis calculation, in that we set $\mathcal{P}_{\zeta}=0$ for the scales on which the model predicts a spectrum greater than the $\mathrm{PBH}$ bound. We have engineered the models so that after $N e$-folds of inflation, our spectrum is just below the PBH bound and that no more perturbations are produced afterwards. In the case of the hilltop-model, we calculate the induced gravitational wave spectrum for $N=55, N=60$, and $N=65$, which we have chosen to reflect the standard choices that appear in the literature, with the lower values of $N$ indicating a lower reheat temperature; an issue which we are investigating 
in a follow up paper. In the case of the running mass model, we select model parameters which both satisfy $n_{s}=0.96$ and $n_{s}^{\prime}=0.002$ and $n_{s}^{\prime}=0.005$ respectively as well as the model parameters predicting the production of PBHs within the Dark Matter range, corresponding to $0.0067<n_{s}^{\prime}<0.012$. Unlike in the hilltop model, no demand for the maximisation of the spectrum at a particular $e$-fold is made. Instead, if the spectrum touches the PBH bound, we terminate inflation, as can be seen in Fig. (6).

We plot the results of Eq. (??) for the induced gravitational waves from the hilltop model in Figures (7) and (8) and for the running mass model in Fig. (10). We also plot the sensitivity curves from the various gravitational wave detectors, LIGO [61], LCGT [62], LISA[29] ${ }^{5}$, BBO/DECIGO [30], cross-correlated DECIGO and Ultimate DECIGO as well as rough estimates of the current pulsar timing limit and the expected limit from the Square Kilometre Array (SKA) [64-67]. We highlight the fact that our estimate for the cross-correlated DECIGO is rather basic by plotting it differently to BBO/DECIGO and ultimate DECIGO. How these curves are generated and the data sets used are explained in Appendix D. The primordial gravitational wave spectrum can also be large enough to be detectable by BBO and DECIGO, as was shown for single large field models of inflation (for example see Refs. [30, 6870]). However, this fact does not affect our conclusions since priomordial gravitational waves predict a scale invariant spectrum, and has been shown here and in previous works (see for example Refs. $[25,26]$ ) and in this paper, the spectrum of induced gravitational waves is not as simple, and hence are distinguishable from each other.

We find that the hilltop model with integral self coupling powers $p=2$ and $q=3$ generates a GW spectrum detectable by the BBO/DECIGO experiment for inflation lasting a reasonable number of $e$-folds. Furthermore, we find that to be detectable by the $\mathrm{BBO} / \mathrm{DECIGO}$ experiment, for inflation terminating within $55 e$-folds, the hilltop model requires coupling powers of $p=2$ and $2.3 \lesssim q \leq 3$ while for $N=60$ the range $q$ is reduced to $2.5 \lesssim q \leq 3$. In the former case $q=2.2$ comes within range of the cross-correlated DECIGO and for the latter $q=2.3$ comes within its range. It can be clearly seen in Figures (7), (8) and (9) that within the sensitivity range of gravitational wave detectors, the results for the $N=60$ and the $N=65$ cases are the same, as expected.

For the running mass model the $n_{s}^{\prime}=0.005$ model requires that inflation terminates after $43 e$-folds of inflation, which is rather difficult to motivate, but predicts an induced gravitational wave signal well within the range of BBO/DECIGO. The parameters which lead to PBH candidates for Dark Matter come within the range of LISA as well as BBO/DECIGO, but they also require an early termination of inflation, between $29 \lesssim N \lesssim 39$. Since these parameter choices predict $V_{0}$ to be on the GUT scale, we can only reduce $N$ by assuming a matter dominated phase of reheating and a reheat temperature of $T_{R H} \sim 1 \mathrm{MeV}$. However, this can be problematic for the induced gravitational wave prediction, since the scales of interest are so small, they re-enter the horizon immediately after the end of inflation. As we mention in the introduction, and several times thereafter, we assume a radiation dominated universe during the formation of these gravitational waves. Including an early matter dominated phase would affect the predictions for the smallest of scales, making our results for the running mass model at the largest $k$ values for $n_{s}^{\prime}=0.005$, as well as the other parameter values which fall within the green shaded region of Fig. (10), questionable. On the other hand $N=65$ is an acceptable value and is compatible with instant reheating into a radiation

\footnotetext{
${ }^{5}$ We use the detector parameters of the original NASA/ESA experiment which are available, however this is now a European only experiment [63] and the detector specificiations have changed slightly, as dicussed in Appendix (D).
} 


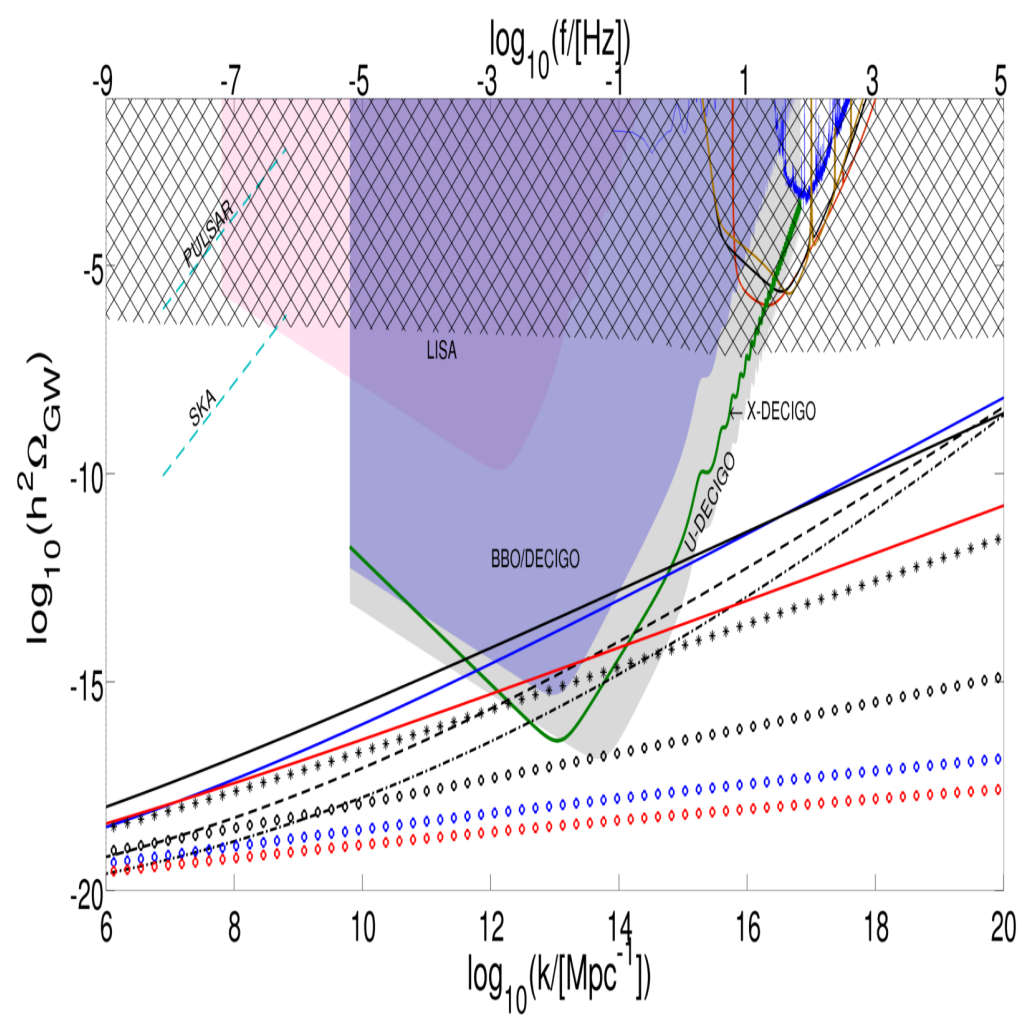

Figure 7. Energy densities, Eq. (2.3), of the induced gravitational wave spectra from hilltop inflation with self-coupling powers labelled in the legend next to Fig. (9). The plot corresponds to maximising the first order spectrum at $N=55$. The shaded regions correspond to the regions of sensitivity of the gravitational wave detectors, with U-DECIGO corresponding to the Ultimate DECIGO detector. The dashed blue lines correspond to the sensitivity from the pulsar timing array. The thick solid green line corresponds to the cross-correlated DECIGO detector (X-DECIGO). The thin straight lines in the upper right hand corner of the plots correspond to Advanced LIGO (red), the 6th run of LIGO (blue), the LCGT official data (black) and the LCGT updated data (brown). The cross-hatched region is the $\mathrm{PBH}$ constraint on the induced gravitational waves from Inflation.

dominated universe. Unfortunately, the spectra for induced gravitational waves for running mass models terminating between $N=51$ and $N=65 e$ - folds do not seem to be within the sensitivity range of future GW detectors, despite the fact that they do result in the formation of PBHs. It may be necessary to asses the impact a matter dominated reheating phase ${ }^{6}$ could have on the eventual induced spectrum of gravitational waves [72, 73, 75, 76].

We should note here that Ref. [43] have recently used the COBE-FIRAS data to further constrain the spectrum on $k<10^{4} \mathrm{Mpc}^{-1}$. They also conclude that the upcoming PIXIE experiment will further constrain this small scale spectrum, which in turn could rule out the running mass model.

\footnotetext{
${ }^{6}$ One can assume other equations of state for this epoch, for example see Refs. [71-74].
} 


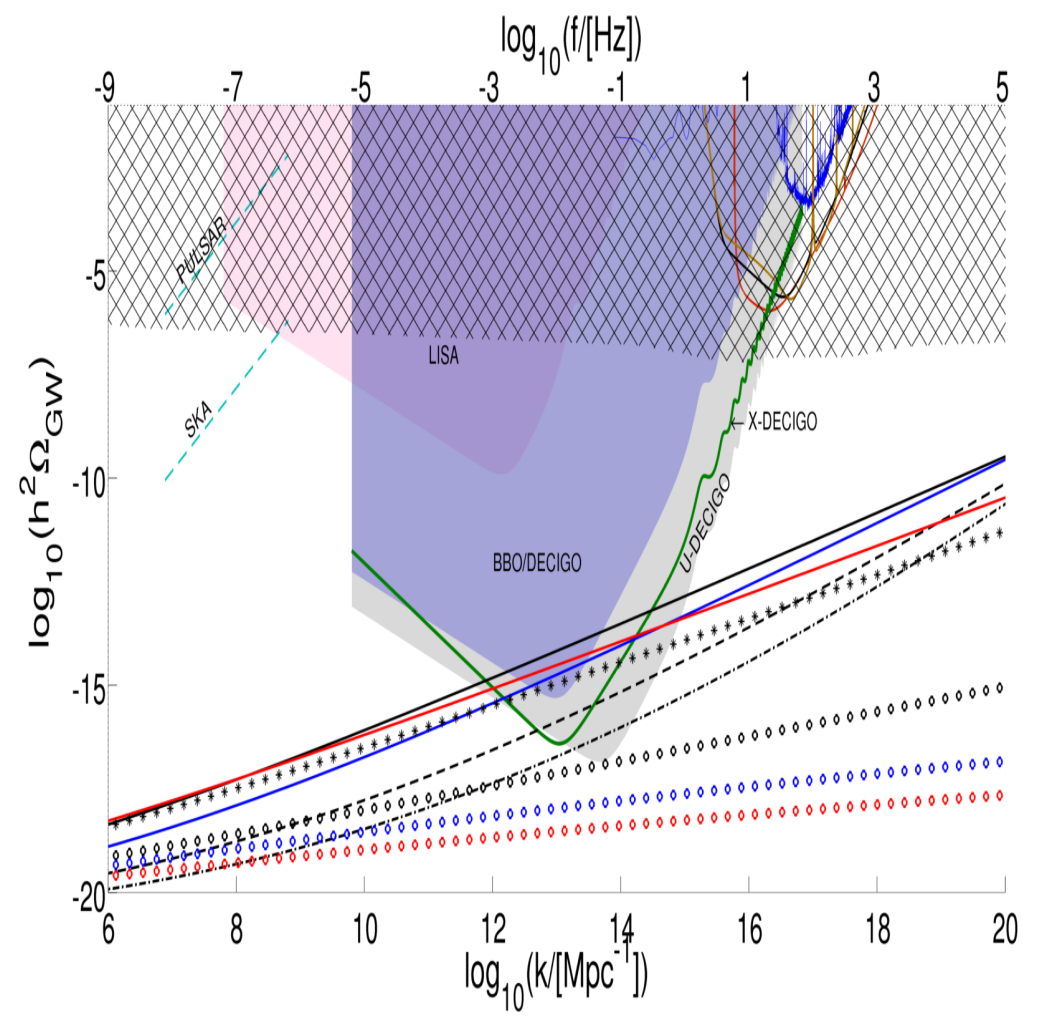

Figure 8. Energy densities, Eq. (2.3), of the induced gravitational wave spectra from hilltop inflation with self-coupling powers labelled in the legend next to Fig. (9). The plot corresponds to maximising the first order spectrum at $N=60$. 


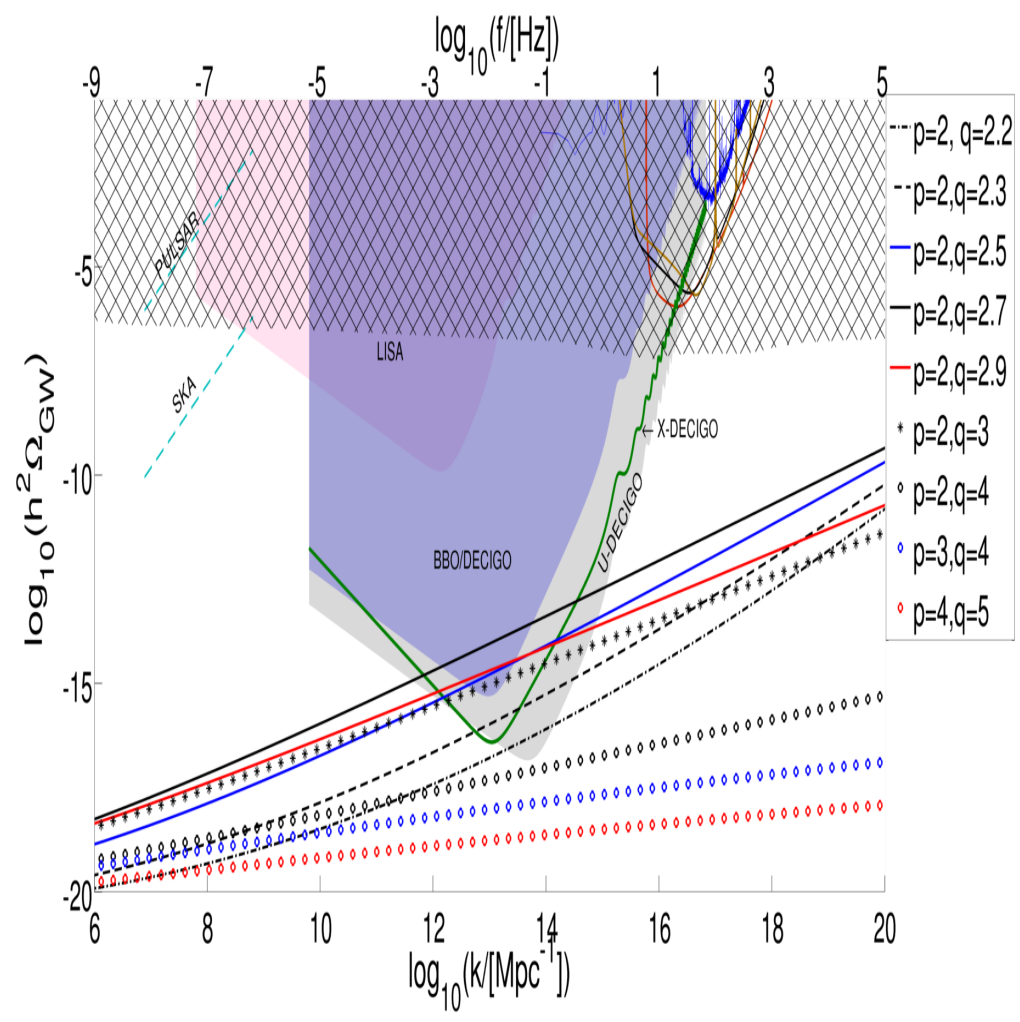

Figure 9. The induced gravitational wave spectrum, Eq. (2.3), for the hilltop model with a scalar spectrum maximised at $N=65 e$-folds. The unlabelled lines in the figure are defined in Fig. (7) 


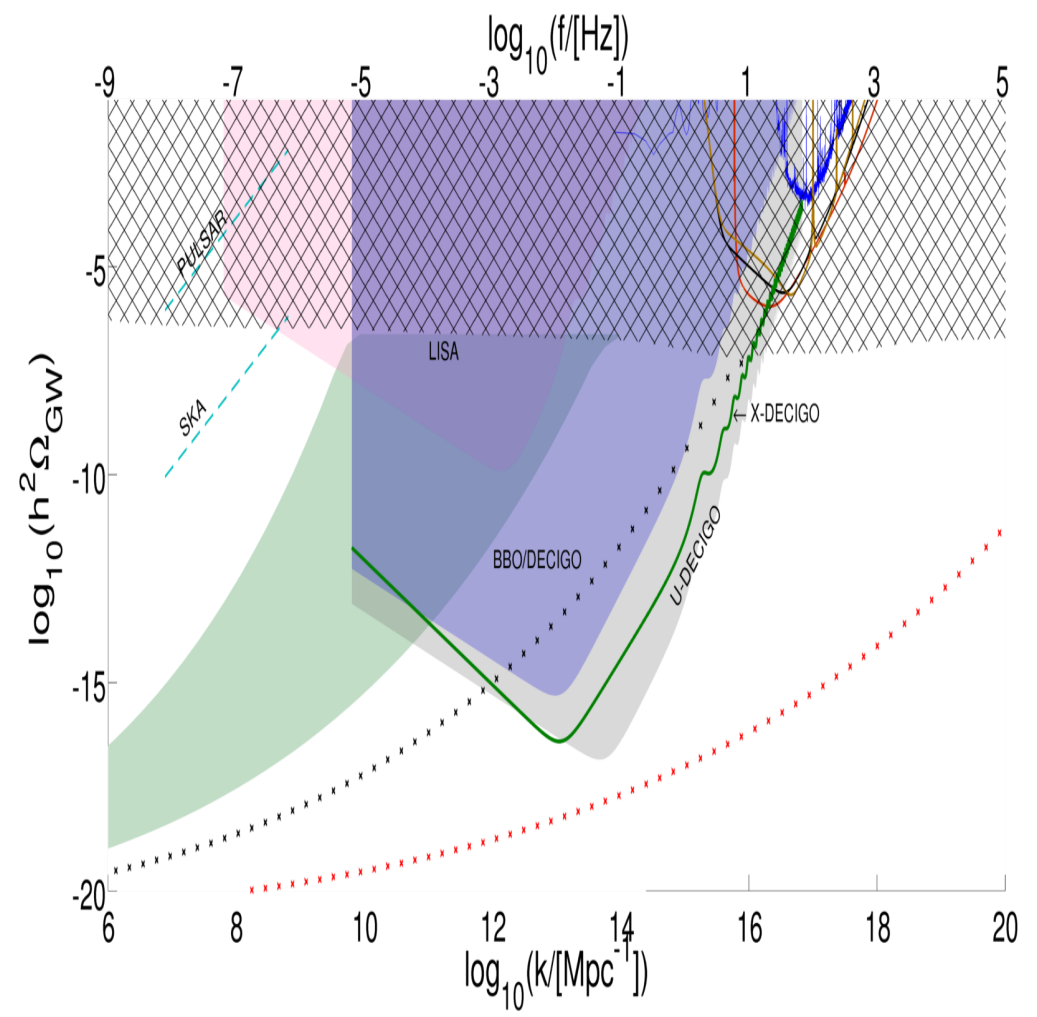

Figure 10. Running mass model predictions for the induced gravitational wave spectra, Eq. (2.3). The $n_{s}^{\prime}=0.002$ (red crosses) model is evolved to $N \sim 64$ while the $n_{s}^{\prime}=0.005$ (black crosses) model requires that inflation terminates at $N \sim 43$ to satisfy the $\mathrm{PBH}$ bound. The green shaded region corresponds to the range of parameters in which result in the production of PBHs whose energy density agrees with that of Dark Matter, and inflation is required to terminate between $29 \lesssim N \lesssim 39$ to satisfy the PBH bound. The hatched region and upper right hand corner lines are all defined in Fig. (9). 


\section{Conclusions}

We have found that for a reasonable range of $e$-folds, Hilltop-type models predict a spectrum of induced gravitational waves likely to be detectable by both the DECIGO and cross correlated DECIGO. More interestingly, the model with integral coupling powers $p=2, q=3$, which is strongly motivated in particle physics models, is within the range of the detection, while satisfying the PBH bounds and WMAP constraints. This is shown in Figures (7), (8) and (9).

On the other hand, the running mass model may also lead to a spectrum within the range of the LISA and DECIGO experiment, if small values of $N$ can be motivated. In which case, the produced PBHs, with masses within the range $M_{\mathrm{BH}} \sim 10^{20}-10^{27} \mathrm{~g}$, can be candidates for dark matter. This scenario will be checked by the future gravitational wave observations (See Fig.10).

We hope the earliest possible completion of these new types of gravitational wave observatories.

\section{Acknowledgments}

We thank A. Christopherson, D. Galliano, T. Hiramatsu, D.H. Lyth, R. Saito, A. Taruya, T. Tanaka, and J. Yokoyama for useful discussions. This work was supported in part by grantin-aid from the Ministry of Education, Culture, Sports, Science, and Technology (MEXT) of Japan, No. 2200775 (L.A.), No. 21111006, No. 23540327, No.22244030 (K.K.). L.A. is also supported by the Japanese Society for the Promotion of Science (JSPS). M.S. acknowledges Monbukagaku-sho Grant-in-Aid for the Global COE programs, The Next Generation of Physics, Spun from Universality and Emergence at Kyoto University, and JSPS Grant-inAid for Scientific Research (A) No. 21244033. K.K. was partly supported by the Center for the Promotion of Integrated Sciences (CPIS) of Sokendai, No. 1HB5806020.

\section{A Induced Gravitational Waves}

In this section we will briefly review the equations relevant to the formation and evolution of gravitational waves sourced by the primordial scalar perturbations [25, 26, 32]. These induced gravitational waves are in essence a physical manifestation of the spatial perturbations which arise from taking the Taylor expansion of the metric up to order second. The ADM metric in this case is given as

$d s^{2}=a^{2}(\tau)\left[-\left(1+2 \Phi^{(1)}+2 \Phi^{(2)}\right) d \tau^{2}+2 V_{i}^{(2)} d \tau d x^{i}+\left\{\left(1-2 \Phi^{(1)}-2 \Phi^{(2)}\right) \delta_{i j}+\frac{1}{2} h_{i j}\right\} d x^{i} d x^{j}\right]$

where we have chosen a longitudinal gauge and assume $\Phi=\Psi$ at all orders, $\Phi$ is the Bardeen potential, first order vector perturbations are ignored $\left(V^{(1)}=0\right)$ and the tensor perturbations $\left(h_{i j}\right)$ include both first and second order effects. In this paper we neglect anisotropic stress. The next step is to calculate the Einstein equations[26, 77]:

$$
\begin{aligned}
G_{j}^{(2) i}= & a^{-2}\left[\frac{1}{4}\left(h_{j}^{i^{\prime \prime}}+2 \mathcal{H} h_{j}^{i^{\prime}}-\nabla^{2} h_{j}^{i}\right)+4 \Phi^{(1)} \partial^{i} \partial_{j} \Phi^{(1)}+2 \partial^{i} \Phi^{(1)} \partial_{j} \Phi^{(1)}\right. \\
& +[\text { Second Order Terms }]+[\text { diagonal terms }]]
\end{aligned}
$$


where the SecondOrderTerms refer to terms containing second order scalar perturbations. The spatial part of the energy-momentum tensor is:

$$
T_{j}^{(2) i}=\left(\rho^{(0)}+P^{(0)}\right) v^{(1) i} v_{j}^{(1)}+P^{(2)} \delta_{j}^{i}
$$

where $\rho$ and $P$ are the energy density and pressure, and $v$ is the velocity. Here on, for simplicity, our notation is such that $h_{i j}=h_{i j}^{(2)}$.

As is standard, to evaluate the spectrum of these gravitational waves the Fourier mode of the tensor perturbation is taken:

$$
h_{i j}(\mathbf{x}, \tau)=\frac{1}{(2 \pi)^{3 / 2}} \int d^{3} k e^{i \mathbf{k} \cdot \mathbf{x}}\left[h_{\mathbf{k}}(\tau) \mathrm{e}_{i j}(\mathbf{k})+\bar{h}_{\mathbf{k}}(\tau) \overline{\mathrm{e}}_{i j}(\mathbf{k})\right]
$$

where the polarization tensors e, $\overline{\mathrm{e}}$ are given in terms of the orthonormal vectors $(\mathbf{e}, \overline{\mathbf{e}})[25$, $26,78]$ :

$$
\mathrm{e}_{i j}(\mathbf{k})=\frac{1}{\sqrt{2}}\left[e_{i} e_{j}-\bar{e}_{i} \bar{e}_{j}\right] \quad \overline{\mathrm{e}}_{i j}(\mathbf{k})=\frac{1}{\sqrt{2}}\left[e_{i} \bar{e}_{j}+\bar{e}_{i} e_{j}\right]
$$

these vectors have been defined to satisfy the conditions that (a) the gravitational waves are traceless, thus $\mathrm{e}_{i j} \delta^{i j}=0,(\mathrm{~b})$ normal and (c) transverse .

According to Refs. [25, 26], there exists a projection tensor defined robustly in Ref. [25], which extracts the transverse, traceless parts of Eq. (A.2) and also does away with the Second Order Terms:

$$
\hat{\mathcal{T}}_{i j}^{l m} G_{l m}^{(2)}=8 \pi G \hat{\mathcal{T}}_{i j}^{l m} T_{l m}^{(2)} .
$$

Thus the equation of motion for the tensor perturbation can be derived, using the following definitions [26]:

$$
\begin{aligned}
P^{(0)}=w \rho^{(0)} & \rho^{(0)}=\frac{3 \mathcal{H}^{2}}{8 \pi G a^{2}} \\
v_{i}^{(1)}= & -\frac{2}{8 \pi G a^{2}(1+w) \rho^{(0)}} \partial_{i}\left(\Phi^{\prime}+\mathcal{H} \Phi\right)
\end{aligned}
$$

$w$ is known as the equation of state, $G$ is the Gravitational constant and we have dropped the superscript $(i)$ from the Bardeen potential and from now on $\Phi$ refers to the first-order Bardeen potential. Therefore from Eqs. (A.2), (A.3) and (A.6), and after some tidying up one gets:

$$
h_{i j}^{\prime \prime}+2 \mathcal{H} h_{i j}^{\prime}-\nabla^{2} h_{i j}=-4 \hat{\mathcal{T}}_{i j}^{l m} \mathcal{S}_{l m}
$$

with the source term:

$$
\mathcal{S}_{i j}=4 \Phi \partial_{i} \partial_{j} \Phi+2 \partial_{i} \Phi \partial_{j} \Phi-\frac{4}{3 \mathcal{H}^{2}(1+w)} \partial_{i}\left(\Phi^{\prime}+\mathcal{H} \Phi\right) \partial_{j}\left(\Phi^{\prime}+\mathcal{H} \Phi\right)
$$

To move to Fourier space, the Fourier transform of the scalar quantity $\Phi$ is written down:

$$
\Phi(\mathbf{x})=\frac{1}{(2 \pi)^{3 / 2}} \int d^{3} k e^{i \mathbf{k} \cdot \mathbf{x}} \Phi_{\mathbf{k}}
$$


terms like $\partial_{l} \Phi$ then will pull down a $i k_{l}$ term and $\partial_{l} \partial_{m} \Phi$ will pull down a $-k_{l} k_{m}$ term, which from Eqs. (A.9) and (A.10) leads to terms of the form:

$$
\begin{aligned}
& \Phi \partial_{l} \partial_{m} \Phi=-\frac{1}{(2 \pi)^{3}} \int d^{3} k e^{i \mathbf{k} \cdot \mathbf{x}}\left[\int d^{3} q q_{l} q_{m} \Phi_{\mathbf{k}-\mathbf{q}} \Phi_{\mathbf{q}}\right] \\
& \partial_{l} \Phi \partial_{m} \Phi=-\frac{1}{(2 \pi)^{3}} \int d^{3} k e^{i \mathbf{k} \cdot \mathbf{x}}\left[\int d^{3} q\left(k_{l}-q_{l}\right) k_{m} \Phi_{\mathbf{k}-\mathbf{q}} \Phi_{\mathbf{q}}\right]
\end{aligned}
$$

The Fourier form of Eq. (A.8) is then:

$$
h_{\mathbf{k}}^{\prime \prime} \mathrm{e}_{i j}+\bar{h}_{\mathbf{k}}^{\prime \prime} \overline{\mathrm{e}}_{i j}+2 \mathcal{H} h_{\mathbf{k}}^{\prime} \mathrm{e}_{i j}+2 \mathcal{H} \bar{h}_{\mathbf{k}}^{\prime} \overline{\mathrm{e}}_{i j}+k^{2} h_{\mathbf{k}} \mathrm{e}_{i j}+k^{2} \bar{h}_{\mathbf{k}} \overline{\mathrm{e}}_{i j}=\cdots
$$

where the $\cdots$ refer to the Fourier transform of the right hand side of Eq. (A.8) and $h_{\mathbf{k}}=h_{\mathbf{k}}(\tau)$. To extract $h$ and get rid of $\bar{h}$ one simply multiplies through by $\mathrm{e}^{i j}(\mathbf{k})$ to get:

$$
\begin{aligned}
h_{\mathbf{k}}^{\prime \prime}+2 \mathcal{H} h_{\mathbf{k}}^{\prime}+k^{2} h_{\mathbf{k}}= & 4 \int \frac{d^{3} q}{(2 \pi)^{3 / 2}} \mathrm{e}^{i j}(\mathbf{k}) \frac{q_{i} q_{j}}{3(1+w)}[ \\
& \left.(10+6 w) \Phi_{\mathbf{q}} \Phi_{\mathbf{k}-\mathbf{q}}+\frac{8}{\mathcal{H}} \Phi_{\mathbf{q}} \Phi_{\mathbf{k}-\mathbf{q}}^{\prime}+\frac{4}{\mathcal{H}^{2}} \Phi_{\mathbf{q}}^{\prime} \Phi_{\mathbf{k}-\mathbf{q}}^{\prime}\right] .
\end{aligned}
$$

To solve this equation, the usual change of variables $a h_{\mathbf{k}}=v_{\mathbf{k}}$ is made, and Eq. (A.13) becomes:

$$
v_{\mathbf{k}}^{\prime \prime}+\left(k^{2}-\frac{a^{\prime \prime}}{a}\right) v_{\mathbf{k}}=a \mathcal{S}
$$

where $\mathcal{S}$ is defined via Eq. (A.13) and is written down explicitly as

$$
a \mathcal{S} \equiv 4 \int \frac{d^{3} q}{(2 \pi)^{3 / 2}} \mathrm{e}^{i j}(\mathbf{k}) \frac{q_{i} q_{j}}{3(1+w)}\left[(10+6 w) \Phi_{\mathbf{q}} \Phi_{\mathbf{k}-\mathbf{q}}+\frac{8}{\mathcal{H}} \Phi_{\mathbf{q}} \Phi_{\mathbf{k}-\mathbf{q}}^{\prime}+\frac{4}{\mathcal{H}^{2}} \Phi_{\mathbf{q}}^{\prime} \Phi_{\mathbf{k}-\mathbf{q}}^{\prime}\right]
$$

The solution of Eq. (A.14) is found via the Greens' function method i.e the solution is written down as:

$$
h_{\mathbf{k}}(\tau)=\frac{1}{a(\tau)} \int d \tilde{\tau} g_{\mathbf{k}}(\tau ; \tilde{\tau})[a(\tilde{\tau}) \mathcal{S}(\mathbf{k}, \tilde{\tau})]
$$

the Greens function is then the solution of:

$$
g_{\mathbf{k}}^{\prime \prime}+\left(k^{2}-\frac{a^{\prime \prime}}{a}\right) g_{\mathbf{k}}=\delta(\tau-\tilde{\tau}) .
$$

To solve Eq. (A.17) the functional form of the scale factor is needed, and hence the epoch should be defined. Therefore $g$ has two forms, one for matter domination (MD) and another for radiation domination (RD). The derivation of which can be found in Appendix A of Ref. [26], here we just state the results:

$$
\begin{aligned}
& g_{k}(\tau ; \tilde{\tau})=\frac{1}{k} \sin [k(\tau-\tilde{\tau})] \\
& g_{k}(\tau ; \tilde{\tau})=-\frac{x \tilde{x}}{k}\left[j_{1}(x) y_{1}(\tilde{x})-j_{1}(\tilde{x}) y_{1}(x)\right]
\end{aligned}
$$


where Eq. (A.18) is for RD, Eq. (A.19) is for MD, $x=k \tau$ and $j_{1}, y_{1}$ are the spherical Bessel functions.

In order to evaluate the source term, the expressions for the Bardeen potential are also required. Since it is a first order quantity it is obtained from linear theory [79],

$$
\Phi_{\mathbf{k}}^{\prime \prime}+\frac{6(1+w)}{1+3 w} \frac{1}{\tau} \Phi_{\mathbf{k}}^{\prime}+w k^{2} \Phi_{\mathbf{k}}=0
$$

and can be solved exactly using Bessel functions:

$$
\Phi_{\mathbf{k}}(\tau)=\tilde{y}^{-\alpha}\left[C_{1}(k) J_{\alpha}(\tilde{y})+C_{2}(k) Y_{\alpha}(\tilde{y})\right]
$$

where $w>0$ and

$$
\tilde{y}=\sqrt{w} k \tau \quad \alpha=\frac{1}{2}\left(\frac{5+3 w}{1+3 w}\right) .
$$

For Matter Domination $w=0$ and:

$$
\Phi_{\mathbf{k}}(\tau)=C_{1}(k)
$$

where we drop the decaying mode, and during Radiation Domination

$$
\Phi_{\mathbf{k}}(\tau)=\frac{1}{\tilde{y}^{2}} C_{1}(k)\left(\frac{\sin (\tilde{y})}{\tilde{y}}-\cos (\tilde{y})\right) .
$$

In the limit of small $\tilde{y}$ (early times) this function reduces to $C_{1}(k)$ and at early times it should be equal to the vacuum perturbation $\psi_{\mathbf{k}}, \Phi_{\mathbf{k}}(\tau \rightarrow 0) \rightarrow \psi_{\mathbf{k}}$. Then we can write:

$$
\Phi_{\mathbf{k}}(\tau)=\psi_{\mathbf{k}} \Phi(k \tau)
$$

where the $\Phi$ on the right hand side is the transfer function which evolves the primordial fluctuation $\psi_{\mathbf{k}}$, and we hope the notational degeneracy is acceptable to the reader.

The main aim is to calculate the spectrum of induced gravitational waves, which is essentially a measure of the correlation between two modes:

$$
<h_{\mathbf{k}} h_{\mathbf{k}^{\prime}}>=\frac{2 \pi^{2}}{k^{3}} \delta\left(\mathbf{k}+\mathbf{k}^{\prime}\right) P_{h}(k, \tau)
$$

and all the ingredients needed to evaluate this spectrum are now in place. The spectrum of Eq. (A.16) is

$$
<h_{\mathbf{k}} h_{\mathbf{k}^{\prime}}>=\frac{1}{a^{2}} \int_{0}^{\tau} d \tau_{1} d \tau_{2} g_{k}\left(\tau ; \tau_{1}\right) g_{k}\left(\tau ; \tau_{2}\right) a\left(\tau_{1}\right) a\left(\tau_{2}\right)<\mathcal{S}\left(\tau_{1}, \mathbf{k}\right) \mathcal{S}\left(\tau_{2}, \mathbf{k}\right)>.
$$

and in this work we are only interested in the RD part. Before we piece things together, we make a small redefinition: $\mathrm{e}(\mathbf{k}, \mathbf{q})=\mathrm{e}^{i j}(\mathbf{k}) q_{i} q_{j}=q^{2}\left(1-\mu^{2}\right)$ where $\mu=(\mathbf{k} \cdot \mathbf{q}) /(k q)$.

The source term can be written as:

$$
\mathcal{S}(\mathbf{k}, \tau)=\frac{1}{(2 \pi)^{3 / 2}} \int d^{3} q \mathrm{e}(\mathbf{k}, \mathbf{q}) f(\mathbf{k}, \mathbf{q}, \tau) \psi_{\mathbf{q}} \psi_{\mathbf{k}-\mathbf{q}}
$$

where

$$
f(\mathbf{k}, \mathbf{q}, \tau)=\frac{2(5+3 w)}{3(1+w)} \Phi_{\mathbf{q}} \Phi_{\mathbf{k}-\mathbf{q}}+\frac{8}{3 \mathcal{H}(1+w)} \Phi_{\mathbf{q}} \Phi_{\mathbf{k}-\mathbf{q}}^{\prime}+\frac{4}{3 \mathcal{H}^{2}(1+w)} \Phi_{\mathbf{q}}^{\prime} \Phi_{\mathbf{k}-\mathbf{q}}^{\prime} .
$$


The primordial spectrum of linear scalar perturbations is given by:

$$
<\psi_{\mathbf{q}} \psi_{\mathbf{k}}>=\frac{2 \pi^{2}}{q^{3}} P(q) \delta(\mathbf{q}+\mathbf{k})
$$

Thus the correlation between two modes of the source term can finally be written as:

$$
\begin{aligned}
<\mathcal{S}\left(\mathbf{k}, \tau_{1}\right) \mathcal{S}\left(\mathbf{k}^{\prime}, \tau_{2}\right)>= & \delta\left(\mathbf{k}+\mathbf{k}^{\prime}\right) \int d^{3} \tilde{k} \mathrm{e}(\mathbf{k}, \tilde{\mathbf{k}})^{2} f\left(\mathbf{k}, \tilde{\mathbf{k}}, \tau_{1}\right)\left[f\left(\mathbf{k}, \tilde{\mathbf{k}}, \tau_{2}\right)\right. \\
& \left.+f\left(\mathbf{k}, \mathbf{k}-\tilde{\mathbf{k}}, \tau_{2}\right)\right] \frac{P(|\mathbf{k}-\tilde{\mathbf{k}}|)}{|\mathbf{k}-\tilde{\mathbf{k}}|^{3}} \frac{P(\tilde{k})}{\tilde{k}^{3}}
\end{aligned}
$$

and the spectrum of induced gravitational waves is:

$$
P_{h}(k)=\frac{1}{a^{2}} \int_{0}^{\infty} d \tilde{k} \int_{-1}^{1} d \mu \frac{k^{3} \tilde{k}^{3}}{|\mathbf{k}-\tilde{\mathbf{k}}|^{3}}\left(1-\mu^{2}\right)^{2} P(|\mathbf{k}-\tilde{\mathbf{k}}|) P(\tilde{k}) I_{1}(k, \tilde{k}, \tau) I_{2}(k, \tilde{k}, \tau)
$$

where

$$
\begin{aligned}
& I_{1}(k, \tilde{k}, \tau)=\int d \tau_{1} a\left(\tau_{1}\right) g_{k}\left(\tau ; \tau_{1}\right) f\left(k, \tilde{k}, \tau_{1}\right) \\
& I_{2}(k, \tilde{k}, \tau)=\int d \tau_{2} a\left(\tau_{2}\right) g_{k}\left(\tau ; \tau_{2}\right)\left[f\left(k, \tilde{k}, \tau_{2}\right)+f\left(k,|\mathbf{k}-\tilde{\mathbf{k}}|, \tau_{2}\right)\right]
\end{aligned}
$$

are generic integrals, and depend only on the epoch of evaluation. We present their results in the next section for radiation domination. First we rewrite the spectrum in terms of the variables $v=\tilde{k} / k, y=\sqrt{1+v^{2}-2 v \mu}$, and $x=k \tau$. During RD $a \propto \tau$ and the spectrum reduces to

$$
P_{h}(k)=\frac{k^{2}}{x^{2}} \int_{0}^{\infty} d v \int_{|v-1|}^{|v+1|} d y \frac{v^{2}}{y^{2}}\left(1-\mu^{2}\right)^{2} P(k v) P(k y) \tilde{I}_{1} \tilde{I}_{2}
$$

where

$$
\begin{aligned}
I_{1}(k, \tilde{k}, \tau) & =\frac{1}{k^{2}} \tilde{I}_{1} \\
& =\frac{1}{k^{2}} \int d x_{1} x_{1} g_{k}\left(x ; x_{1}\right) f\left(k, x_{1} v\right) \\
I_{2}(k, \tilde{k}, \tau) & =\frac{1}{k^{2}} \tilde{I}_{2} \\
& =\frac{1}{k^{2}} \int d x_{2} x_{2} g_{k}\left(x ; x_{2}\right)\left[f\left(k, x_{2} v\right)+f\left(k, x_{2} y\right)\right]
\end{aligned}
$$

\section{A.1 The $\tau$ integrals}

This was first shown in Ref. [25]. The first integral in Eq. (A.35) is found to be:

$$
\begin{aligned}
\tilde{I}_{1}= & \frac{1}{4 k y^{3} v^{3}}\left\{-\cos (x) \sum_{n=1}^{4} \alpha_{n} \operatorname{Si}\left(\beta_{\mathrm{n}} \mathrm{x}\right)+\sin (\mathrm{x}) \sum_{\mathrm{n}=1}^{4}(-1)^{\mathrm{n}+1} \alpha_{\mathrm{n}} \mathrm{ci}\left(\beta_{\mathrm{n}} \mathrm{x}\right)\right\} \\
& +\gamma_{1} \sin (x)+\gamma_{2} \sin (v x) \sin (y x)+\gamma_{3} \sin (v x) \cos (y x)+\gamma_{4} \cos (v x) \sin (y x)+\gamma_{5} \cos (v x) \cos (y x)
\end{aligned}
$$


and the second integral is given by:

$$
\begin{aligned}
\tilde{I}_{2}= & -\frac{\alpha}{2 k v^{3} y^{3}}\left\{\cos (x)\left[-\operatorname{Si}\left(\beta_{1} \mathrm{x}\right)+\operatorname{Si}\left(\beta_{2} \mathrm{x}\right)+\operatorname{Si}\left(\beta_{3} \mathrm{x}\right)-\operatorname{Si}\left(\beta_{4} \mathrm{x}\right)\right]\right. \\
& \left.+\sin (x)\left[\operatorname{ci}\left(\beta_{1} \mathrm{x}\right)+\operatorname{ci}\left(\beta_{2} \mathrm{x}\right)-\operatorname{ci}\left(\beta_{3} \mathrm{x}\right)-\operatorname{ci}\left(\beta_{4} \mathrm{x}\right)\right]\right\} \\
& +\gamma_{21} \sin (x)+\gamma_{22} \sin (x(v+y))+\gamma_{23} \sin (x(v-y))+\gamma_{24} \cos (x(v-y))+\gamma_{25} \cos (x(v+y))
\end{aligned}
$$

the coefficients in these two integrals are given in Appendix B. The $\alpha_{n}$ coefficients in the first integral are found to have the property that $\sum_{n=1}^{4}(-1)^{n} \alpha_{n}=0$ and the $\alpha$ coefficient in the second integral is given by $\alpha=\left(v^{2}-1+y^{2}\right)^{2}$.

The Si and ci terms are the sine and cosine integrals respectively [80], defined as:

$$
\begin{aligned}
\operatorname{Si}(\mathrm{x}) & =\int_{0}^{x} \frac{\sin (t)}{t} d t \\
\mathrm{ci}(\mathrm{x}) & =\int_{0}^{x} \frac{\cos (t)}{t} d t .
\end{aligned}
$$

In the limit of large $x$ the sine integral asymptotes to a constant value $S i(x) \rightarrow\left(1+\gamma_{\text {euler }}\right)$ and the cosine integral asymptotes to $c i(x) \rightarrow \ln \left(x / \gamma_{\text {euler }}\right)$, where $\gamma_{\text {euler }}$ is the Euler-Mascheroni constant. The integrals are found to asymptote to 0 for large $v$ and $y$, as well as for when $v, y$ or $x$ approach 0. These are useful properties, and it means that Eq. (A.34) need not be evaluated for $v \rightarrow \infty$ but to a much smaller value, and the final results will be the same. We plot the properties of these integrals in Fig. (11), which shows agreement between our numerics and those of Refs. [25] and [33].

\section{A.2 The energy density of second order gravitational waves}

Detectors of gravitational waves will measure the amplitude of their energy density which can be parametrized by the dimensionless variable (e.g. Ref. [81]):

$$
\Omega_{\mathrm{GW}}(f)=\frac{1}{\rho_{c}} \frac{d \rho_{\mathrm{GW}}}{d \ln f}
$$

where $f$ is the frequency, $\rho_{c}$ is the critical energy density defining the coasting solution of the Friedman equation and $\rho_{\mathrm{GW}}$ is the energy density of gravitational waves. This parameter is related to the spectrum of induced gravitational waves $P_{h}$ as [26]

$$
\Omega_{\mathrm{GW}}^{(2)}=\frac{a(\tau)}{a_{\mathrm{eq}} k_{\mathrm{eq}}^{2}} t^{2}(k, \tau) P_{h}(k, \tau)
$$

where $e q$ denotes matter-radiation equality, and $t$ is the transfer function found to be [26]

$$
t(k, \tau)=\frac{a_{\mathrm{eq}} k_{\mathrm{eq}}}{a(\tau) k}
$$

for scales $k \gg k_{\text {eq }}$, our regime of interest and $k_{\text {eq }} \simeq 0.01 \mathrm{Mpc}^{-1}$. This reduces Eq. (A.40) to:

$$
\Omega_{\mathrm{GW}}^{(2)}(k, z)=\frac{(1+z)}{\left(1+z_{\mathrm{eq}}\right)} P_{h}(k)
$$

where we made the $\tau \sim k^{-1}$ substitution, the time when a scale enters the horizon. Thus today $\Omega_{\mathrm{GW}}\left(k, \eta_{0}\right) \simeq \frac{\mathcal{P}_{h}(k)}{3300}$, where $1+z_{\mathrm{eq}} \simeq 3300$. 

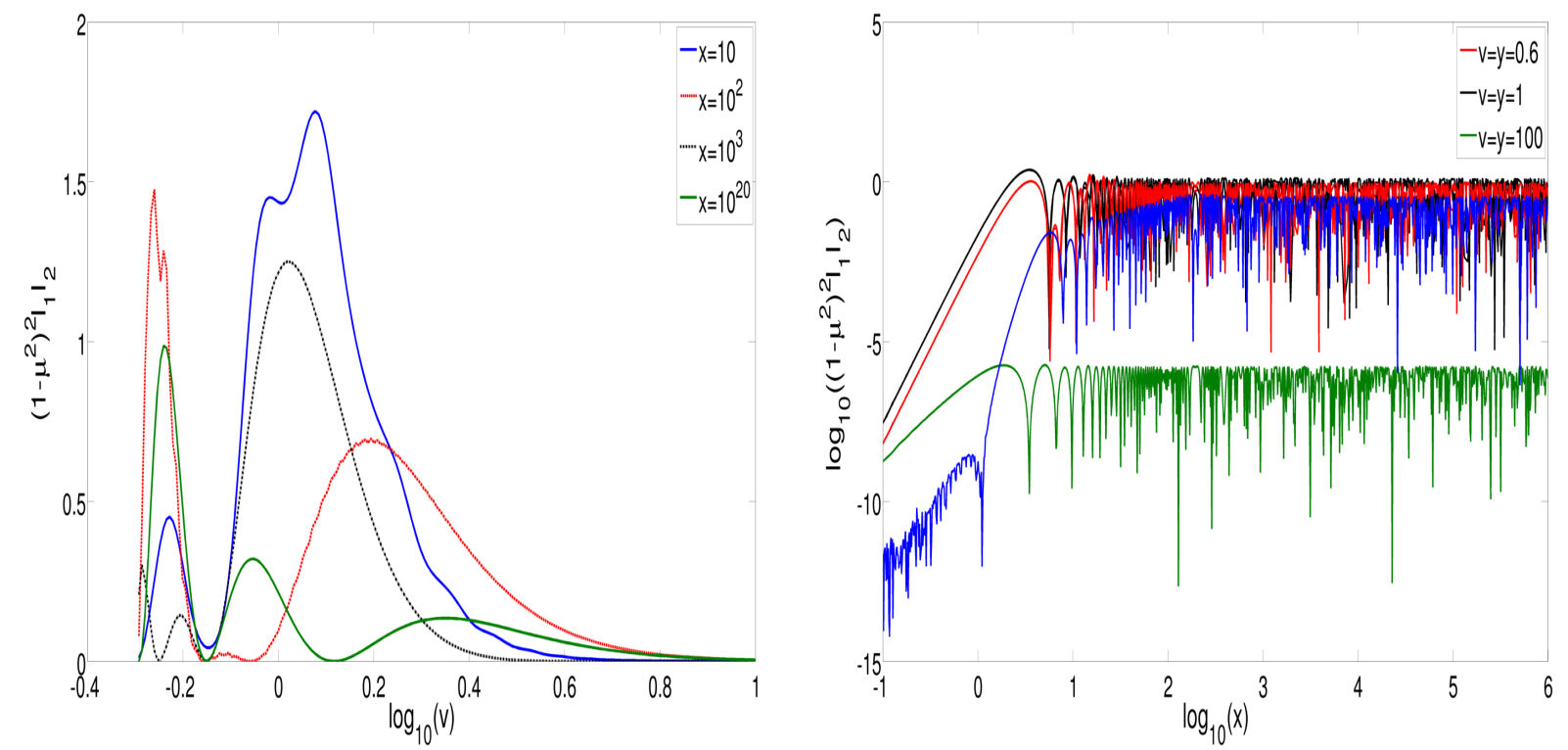

Figure 11. Plot of the $\tau$ integrals for fixed values of $x=k \tau$ and varying $v$ (left), where we have set $y=v$, which clearly shows that the integrals asymptote to zero for large $v$ meaning we need not integrate over an infinite range of scales. Plot of the $\tau$ integrals for fixed $v=y$ and varying $x=k \tau$ (right), this shows that (a) the effect is physical since the source term peaks after horizon entry $(x>1)$ and $(\mathrm{b})$ the envelope of the integrals rapidly asymptotes to a constant value for fixed $v$ and $y$.

\section{B The coefficients of the $\tau$ integral}

We present the coefficients of the time integrals (A.36) and (A.37) in the following tables.

\begin{tabular}{|c|c|c|c|c|}
\cline { 3 - 5 } \multicolumn{2}{|c|}{} & Coefficient & Symbol & Expression \\
\cline { 2 - 5 } \multicolumn{1}{c|}{} & $\sin (x)$ & $\gamma_{1}$ & $\frac{1}{k v^{2} y^{2}}\left(v^{2}-3 y^{2}+1\right)$ \\
$\sin (v x) \sin (y x)$ & $\gamma_{2}$ & $\frac{1}{k x^{3} y^{3} v^{3}}\left(2-x^{2}-x^{2} y^{2}+3 x^{2} v^{2}\right)$ \\
\hline$\beta_{1}$ & $1+v+y$ & $-\frac{2}{k y^{2} x^{2} v^{3}}$ \\
$\beta_{2}$ & $-1+v+y$ & $\sin (v x) \cos (y x)$ & $\gamma_{3}$ & $-\frac{2}{k x^{2} y^{3} v^{2}}$ \\
$\beta_{3}$ & $1+v-y$ & $\cos (v x) \sin (y x)$ & $\gamma_{4}$ & $\frac{2}{k x y^{2} v^{2}}$ \\
$\beta_{4}-1+v-y$ & $\cos (v x) \cos (y x)$ & $\gamma_{5}$ & $\frac{2}{k v^{2} y^{2}}\left(1-v^{2}-y^{2}\right)$ \\
\cline { 2 - 5 } & $\sin (x)$ & $\gamma_{21}$ & $\frac{2}{k x^{2} v^{3} y^{3}}(v-y)$ \\
$\sin (x(v-y))$ & $\gamma_{22}$ & $-\frac{2}{k x^{2} v^{3} y^{3}}(v+y)$ \\
$\sin (x(v+y))$ & $\gamma_{23}$ & $\frac{1}{k x^{2}}\left(-2+x^{2}-x^{2} y^{2}-x^{2} v^{2}-2 x^{2} v y\right)$ \\
$\cos (x(v-y))$ & $\gamma_{24}$ & $-\frac{1}{k x^{3} y^{3} v^{3}}\left(2-x^{2}+x^{2} y^{2}+x^{2} v^{2}-2 x^{2} v y\right)$ \\
\hline
\end{tabular}

Table 2. Table on the left gives the expressions for the coefficients of the arguments of the Cosine and Sine integrals in Eqs. (A.36) and (A.37). The right table gives the expressions for the $\tau_{1}$ integral Eq. (A.36) (tob block) and for the $\tau_{2}$ integral Eq. (A.37) (bottom block). 


\begin{tabular}{|c|c|c|c|c|c|c|c|}
\hline & 1 & $v^{4}$ & $4 v^{3}$ & $4 v^{2}$ & $3 y^{4}$ & $4 y^{3}$ & $2 y^{2} v^{2}$ \\
\hline$\alpha_{1}$ & - & + & + & + & - & - & - \\
$\alpha_{2}$ & + & - & + & - & + & - & + \\
$\alpha_{3}$ & + & - & - & - & + & - & + \\
$\alpha_{4}$ & - & + & - & + & - & - & - \\
\hline
\end{tabular}

Table 3. This table gives the expressions of the coefficients of the sine and cosine integrals in Eq. (A.36). Each $\alpha_{n}$ coefficient has the same parameters as the others, but the parameters differ in their respective signs. The columns to the right of the $\alpha$ s give the sign of the parameter defined in the column header. For example then we can read off $\alpha_{1}$ as $-1+v^{4}+4 v^{3}+4 v^{2}-3 y^{4}-4 y^{3}-2 y^{2} v^{2}$.

\section{The coefficients of the Hilltop Model}

In this section we list the values of $\eta_{p}$ and $\eta_{q}$ for the various coupling powers $p$ and $q$ in the hilltop model, Eq. (5.1). In each case the model parameters are chosen to satisfy $n_{s}=0.95$, and the selection criteria is explained fully in Section 5.1. We draw the readers attention to the horizontal dashed line in the tables, this line separates the fractional powers from the integral powers, and it is clear that for the integral powers, the mass coupling parameters are most strongly constrained by the bound on the spectral index and not from the maximisation of spectrum at the end of inflation.

\begin{tabular}{|c|c|c|c|c|c|c|}
\hline$p$ & $q$ & $\eta_{p}$ & $\eta_{q}$ & $\mathcal{P}_{\zeta}\left(k_{\text {end }}\right)$ & $n_{s}^{\prime}\left(k_{0}\right)$ & $V_{0}^{1 / 4}$ \\
\hline 2 & 2.2 & 0.17959 & 0.1449 & 0.017069 & 0.0059605 & 0.0014 \\
\hline 2 & 2.3 & 13673 & 0.09898 & 0.014 & 0.0078104 & 0.0015 \\
\hline 2 & 2.5 & & 0.07 & & 0.0 & 0.0015 \\
\hline 2 & 2.7 & 0.08 s & 0.04 & 942 & & 0.0017 \\
\hline 2 & 2.9 & & & & & 0.0015 \\
\hline$\overline{2}$ & $-\overline{3}$ & 0 & $\overline{0} . \overline{0} \overline{3}$ & $\overline{0} . \overline{000}$ & $0 . \overline{0}$ & $\overline{0.00 \overline{1} \overline{5}}$ \\
\hline 2 & 4 & 0.038333 & 0.0083333 & $2.239 e-06$ & 0.016928 & 0.0015 \\
\hline 3 & 4 & 0.041667 & & & 0.016712 & 0.0012 \\
\hline 4 & 5 & 0.0375 & 0.028421 & $7.4549 e-08$ & 0.016752 & 0.0009 \\
\hline
\end{tabular}

Table 4. Values of the coupling masses which satisfy the WMAP bounds at the pivot scale $k_{0}=$ $0.002 \mathrm{Mpc}^{-1}$ and maximise the spectrum at $N=55 \mathrm{e}$-folds. Also included is energy scale of the model evaluated at $k=k_{0}$ in units of $\mathrm{m}_{\mathrm{Pl}}$. 


\begin{tabular}{|c|c|c|c|c|c|c|}
\hline$p$ & $q$ & $\eta_{p}$ & $\eta_{q}$ & $\mathcal{P}_{\zeta}\left(k_{e n d}\right)$ & $n_{s}^{\prime}\left(k_{0}\right)$ & $V_{0}^{1 / 4}$ \\
\hline 2 & 2.2 & 0.16939 & 0.15102 & 0.014497 & 0.0051655 & 0.0008 \\
\hline 2 & 2.3 & .128 & 0.095833 & 0.014173 & 0.0066689 & 0.0013 \\
\hline 2 & 2.5 & 71 & & & 0.010 & 0.0009 \\
\hline 2 & 2.7 & 7 & & & 0.0 & 0.001 \\
\hline 2 & 2.9 & 070 & & & & 0.0016 \\
\hline$\overline{2}$ & $-\overline{3}$ & & 0.0 & $\overline{0} . \overline{0} \bar{c}$ & $0 . \overline{0}$ & $\overline{0.001} \overline{6}$ \\
\hline 2 & 4 & 0.0 & 0.0083333 & 3.777 & 0.016235 & 000.11 \\
\hline 3 & 4 & 041667 & 0.025 & $2.4814 e-07$ & 0.01 & 0.0008 \\
\hline 4 & 5 & 0.039583 & 0.03125 & $8.4841 e-08$ & 0.015931 & 0.0007 \\
\hline
\end{tabular}

Table 5. Values of the coupling masses which satisfy the WMAP bounds at the pivot scale $k_{0}=$ $0.002 \mathrm{Mpc}^{-1}$ and maximise the spectrum at $N=60 \mathrm{e}$-folds. Also included is energy scale of the model evaluated at $k=k_{0}$ in units of $\mathrm{m}_{\mathrm{Pl}}$.

\begin{tabular}{|c|c|c|c|c|c|c|}
\hline$p$ & $q$ & $\eta_{p}$ & $\eta_{q}$ & $\mathcal{P}_{\zeta}\left(k_{\text {end }}\right)$ & $n_{s}^{\prime}\left(k_{0}\right)$ & $V_{0}^{1 / 4}$ \\
\hline 2 & 2.2 & 0.16735 & 0.13878 & 0.20683 & 0.0049911 & 0.0008 \\
\hline 2 & 2.3 & 0.12857 & 0.11633 & 0.15491 & 0.0067744 & 0.0009 \\
\hline 2 & 2.3 & 0.096939 & 0.058163 & c0.070402 & 0.010396 & 0.0012 \\
\hline 2 & 2.7 & 0.085417 & 0.042 & 0.062686 & 0.01505 & 0.0016 \\
\hline 2 & 2.9 & 0.071667 & 0.03125 & 0.0069787 & 0.016301 & 0.0016 \\
\hline$\overline{2}$ & $\overline{3}$ & $0.0 \overline{6} \overline{6}$ & $\overline{0} . \overline{0} \overline{6}^{-}$ & $0.00 \overline{2} 4 \overline{5} \overline{9} 9$ & $\overline{0} . \overline{0} 16 \overline{4} \overline{61}$ & $\overline{0} . \overline{0} 0 \overline{1} \overline{6}$ \\
\hline 2 & 4 & 0.036207 & 0.008 & $5.5689 e-06$ & 0.01517 & 0.0016 \\
\hline 3 & 4 & 0.048276 & 0.034 & $3.1526 e-07$ & 0.016363 & 0.0017 \\
\hline 4 & 5 & 0.026552 & 0.018 & $7.8237 e-08$ & 0.013356 & 0.0017 \\
\hline
\end{tabular}

Table 6. Values of the coupling masses which satisfy the WMAP bounds at the pivot scale $k_{0}=$ $0.002 \mathrm{Mpc}^{-1}$ and maximise the spectrum at $N=65 \mathrm{e}$-folds. Also included is energy scale of the model evaluated at $k=k_{0}$ in units of $\mathrm{m}_{\mathrm{Pl}}$.

\section{The sensitivity curves}

In this paper we plot the sensitivity curves of LIGO [61, 82],LISA [29], LCGT[62], DECIGO $[30,31]$. For the space based detectors we use the online sensitivity curve generator in [83] inputting the detector parameters given in Table $7^{7}$. The sensitivity curve generator produces the strain sensitivity defined as [85],

$$
h_{\mathrm{eff}}(f)=\sqrt{\frac{S_{n}(f)}{\mathcal{N}(f)}},
$$

where the units are in $H z^{-1 / 2}$, and $S_{n}$ is known as the noise spectral density and $\mathcal{N}$ is the interferometer response function. To convert between the strain efficiency and the energy density per logarithmic interval we use $\Omega_{g w}=4 \pi^{2} f^{3} S_{h}(f) /\left(3 H_{0}^{2}\right)$ [85], where $S_{h}$ is the signal spectral density and we can then write this as [81] :

\footnotetext{
${ }^{7}$ The $S_{\text {shot }}$ values for DECIGO and Ultimate DECIGO are the updated values of Ref. [84] kindly provided by the authors in a private communication
} 


\begin{tabular}{|c||c|c|c|c|c|c|}
\hline & $L[\mathrm{~m}]$ & $S_{\text {shot }}\left[\mathrm{mHz}^{-1 / 2} \mid\right]$ & $S_{\text {accel }}\left[\mathrm{ms}^{-2} \mathrm{~Hz}^{-1 / 2}\right]$ & $P[W]$ & $D[\mathrm{~m}]$ & $\lambda[\mathrm{nm}]$ \\
\hline LISA & $5 \times 10^{9}$ & $2 \times 10^{-11}$ & $3 \times 10^{-15}$ & 1 & 0.3 & 1064 \\
BBO/DECIGO & $5 \times 10^{7}$ & $1.1 \times 10^{-16}$ & $7.9 \times 10^{-19}$ & 10 & 1 & 532 \\
Ultimate DECIGO & $5 \times 10^{7}$ & $1.7 \times 10^{-18}$ & $3 \times 10^{-19}$ & 10 & 1 & 532 \\
\hline
\end{tabular}

Table 7. Detector parameters used to input into the sensitivity curve generator in Ref. [83]. Where $S_{\text {shot }}$ is the root spectral position noise budget, $S_{\text {accel }}$ is the root spectral density acceleration noise, $P$ is the power of the laser, $D$ is the telescope/mirror diameter and $\lambda$ is the wavelength. The new ESA only LISA mission has an arm length of $10^{9} \mathrm{~m}$ [63], and the difference in sensitivity is shown in Fig. (12) in terms of the energy density of gravitational waves.

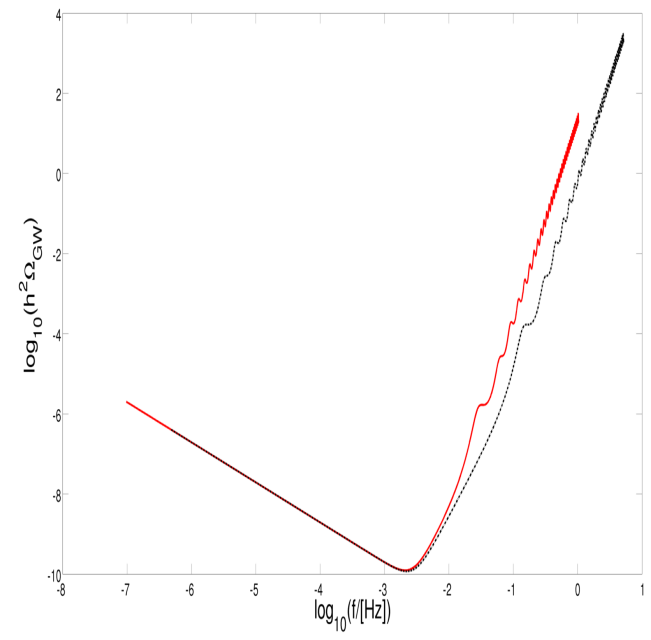

Figure 12. Plot of the energy density of gravitational waves vs. the frequency in Hertz, for the original NASA/ESA LISA mission (red) and the new ESA only eLISA mission (black dash-dot). In this paper we have plotted the sensitivity range of the original LISA mission, and as can be seen from this figure, the difference with the new eLISA mission is negligible for our purposes.

$$
h_{0}^{2} \Omega_{\mathrm{GW}}^{\min }(f) \simeq 0.0125 \times \mathrm{SNR}^{2}\left(\frac{f}{100 \mathrm{~Hz}}\right)^{3}\left(\frac{h_{\mathrm{eff}}}{10^{-22} \mathrm{~Hz}^{-1 / 2}}\right)^{2}
$$

where SNR is the signal to noise ratio, which for interferometer type detectors is given as [81] $\mathrm{SNR}=\sqrt{2 S_{h}(f) / S_{n} f}$.

Finally we also plot what is known as the cross-correlation sensitivity of the BBODECIGO detector. In this case two separate DECIGO detectors are launched and the correlation of their signals results in a significant reduction in the overall sensitivity [86]. The strain efficiency of cross-correlated detectors is given as [85]

$$
h_{\mathrm{eff}, \operatorname{cross}}(f)=\frac{\mathrm{SNR}^{2}}{(2 T \Delta f)^{1 / 4}} \overline{\left(\frac{\left|\mathcal{N}_{12}\right|^{2}}{S_{n 1} S_{n 2}}\right)}(-1 / 4)
$$

where $T$ is the observation time in seconds, $\mathcal{N}_{12}$ is given as $\left.<S_{1} S_{2}\right\rangle=\int_{0}^{\infty} d f S_{h}(f) \mathcal{N}_{12}, \Delta f$ is the frequency resolution, and the overbar is the average over a frequency interval. In this paper we use a rough estimate, we assume that the strain efficiency of each detector is the same and that the detectors are co-aligned and coincident (i.e. that their position vectors are the same). Then the strain efficiency reduces to 


$$
h_{\mathrm{eff}, \mathrm{cross}} \sim \mathrm{SNR}^{2} \frac{h_{\mathrm{eff}}}{(2 T \Delta f)^{1 / 4}}
$$

and in our analysis we take $\Delta f=f / 10$ for the cross correlated DECIGO detector.

\section{References}

[1] WMAP Collaboration, E. Komatsu et al., Seven-Year Wilkinson Microwave Anisotropy Probe (WMAP) Observations: Cosmological Interpretation, Astrophys. J. Suppl. 192 (2011) 18, [arXiv:1001.4538], [doi:10.1088/0067-0049/192/2/18].

[2] SDSS Collaboration, K. N. Abazajian et al., The Seventh Data Release of the Sloan Digital Sky Survey, Astrophys. J. Suppl. 182 (2009) 543-558, [arXiv:0812.0649], [doi:10.1088/0067-0049/182/2/543].

[3] M. Hicken, W. Wood-Vasey, S. Blondin, P. Challis, S. Jha, et al., Improved Dark Energy Constraints from 100 New CfA Supernova Type Ia Light Curves, Astrophys.J. 700 (2009) 1097-1140, [arXiv:0901.4804], [doi:10.1088/0004-637X/700/2/1097].

[4] A. A. Meiksin, The Physics of the Intergalactic Medium, Rev.Mod.Phys. 81 (2009) 1405-1469, [arXiv:0711.3358], [doi:10.1103/RevModPhys.81.1405].

[5] S. Bird, H. V. Peiris, M. Viel, and L. Verde, Minimally Parametric Power Spectrum Reconstruction from the Lyman-alpha Forest, Mon.Not.Roy.Astron.Soc. 413 (2011) 1717-1728, [arXiv:1010.1519], [doi:10.1111/j.1365-2966.2011.18245.x]. * Temporary entry *.

[6] A. Lewis and A. Challinor, Weak gravitational lensing of the cmb, Phys.Rept. 429 (2006) 1-65, [arXiv:astro-ph/0601594], [doi:10.1016/j.physrep.2006.03.002].

[7] E. Komatsu and U. Seljak, The Sunyaev-Zel'dovich angular power spectrum as a probe of cosmological parameters, Mon.Not.Roy.Astron.Soc. 336 (2002) 1256, [arXiv:astro-ph/0205468], [doi:10.1046/j.1365-8711.2002.05889.x].

[8] A. S. Josan and A. M. Green, Gamma-rays from ultracompact minihalos: potential constraints on the primordial curvature perturbation, Phys.Rev. D82 (2010) 083527, [arXiv:1006.4970], [doi:10.1103/PhysRevD.82.083527].

[9] T. Bringmann, P. Scott, and Y. Akrami, Improved constraints on the primordial power spectrum at small scales from ultracompact minihalos, arXiv:1110.2484.

[10] Y. Yang, L. Feng, X. Huang, X. Chen, T. Lu, et al., Constraints on ultracompact minihalos from extragalactic gamma-ray background, JCAP 1112 (2011) 020, [arXiv:1112.6229].

[11] F. Li, A. L. Erickcek, and N. M. Law, A new probe of the small-scale primordial power spectrum: astrometric microlensing by ultracompact minihalos, arXiv:1202.1284.

[12] P. Scott, T. Bringmann, and Y. Akrami, Constraints on small-scale cosmological perturbations from gamma-ray searches for dark matter, arXiv:1205.1432.

[13] B. Carr, K. Kohri, Y. Sendouda, and J. Yokoyama, New cosmological constraints on primordial black holes, Phys.Rev. D81 (2010) 104019, [arXiv:0912.5297], [doi:10.1103/PhysRevD.81.104019].

[14] A. S. Josan, A. M. Green, and K. A. Malik, Generalised constraints on the curvature perturbation from primordial black holes, Phys.Rev. D79 (2009) 103520, [arXiv:0903.3184], [doi:10.1103/PhysRevD.79.103520].

[15] S. M. Leach, I. J. Grivell, and A. R. Liddle, Black hole constraints on the running mass inflation model, Phys. Rev. D62 (2000) 043516, [arXiv:astro-ph/0004296], [doi:10.1103/PhysRevD.62.043516].

[16] K. Kohri, C.-M. Lin, and D. H. Lyth, More hilltop inflation models, JCAP 0712 (2007) 004, [arXiv:0707.3826], [doi:10.1088/1475-7516/2007/12/004]. 
[17] K. Kohri, D. H. Lyth, and A. Melchiorri, Black hole formation and slow-roll inflation, JCAP 0804 (2008) 038, [arXiv:0711.5006], [doi:10.1088/1475-7516/2008/04/038].

[18] L. Alabidi and K. Kohri, Generating Primordial Black Holes Via Hilltop-Type Inflation Models, Phys. Rev. D80 (2009) 063511, [arXiv:0906.1398], [doi:10.1103/PhysRevD.80.063511].

[19] A. S. Josan and A. M. Green, Constraints from primordial black hole formation at the end of inflation, Phys.Rev. D82 (2010) 047303, [arXiv:1004.5347], [doi:10.1103/PhysRevD.82.047303].

[20] S. Matarrese, O. Pantano, and D. Saez, General relativistic dynamics of irrotational dust: Cosmological implications, Phys.Rev.Lett. 72 (1994) 320-323, [arXiv:astro-ph/9310036], [doi:10.1103/PhysRevLett.72.320].

[21] S. Matarrese, S. Mollerach, and M. Bruni, Second order perturbations of the Einstein-de Sitter universe, Phys.Rev. D58 (1998) 043504, [arXiv:astro-ph/9707278], [doi:10.1103/PhysRevD.58.043504].

[22] H. Noh and J.-c. Hwang, Second-order perturbations of the Friedmann world model, Phys.Rev. D69 (2004) 104011, [doi:10.1103/PhysRevD.69.104011].

[23] C. Carbone and S. Matarrese, A Unified treatment of cosmological perturbations from super-horizon to small scales, Phys.Rev. D71 (2005) 043508, [arXiv:astro-ph/0407611], [doi:10.1103/PhysRevD.71.043508].

[24] K. Nakamura, Second-order gauge invariant cosmological perturbation theory: Einstein equations in terms of gauge invariant variables, Prog.Theor.Phys. 117 (2007) 17-74, [arXiv:gr-qc/0605108], [doi:10.1143/PTP.117.17]. Complete version of gr-qc/0605107.

[25] K. N. Ananda, C. Clarkson, and D. Wands, The Cosmological gravitational wave background from primordial density perturbations, Phys.Rev. D75 (2007) 123518, [arXiv:gr-qc/0612013], [doi:10.1103/PhysRevD.75.123518].

[26] D. Baumann, P. J. Steinhardt, K. Takahashi, and K. Ichiki, Gravitational Wave Spectrum Induced by Primordial Scalar Perturbations, Phys.Rev. D76 (2007) 084019, [arXiv:hep-th/0703290], [doi:10.1103/PhysRevD.76.084019].

[27] J. L. Cook and L. Sorbo, Particle production during inflation and gravitational waves detectable by ground-based interferometers, Phys.Rev. D85 (2012) 023534, [arXiv:1109.0022], [doi:10.1103/PhysRevD.85.023534]. 20 pages, 3 figures, version on Phys. Rev. D.

[28] H. Assadullahi and D. Wands, Constraints on primordial density perturbations from induced gravitational waves, Phys.Rev. D81 (2010) 023527, [arXiv:0907.4073], [doi:10.1103/PhysRevD.81.023527].

[29] http://lisa.nasa.gov/.

[30] N. Seto, S. Kawamura, and T. Nakamura, Possibility of direct measurement of the acceleration of the universe using 0.1- $\mathrm{Hz}$ band laser interferometer gravitational wave antenna in space, Phys.Rev.Lett. 87 (2001) 221103, [arXiv:astro-ph/0108011], [doi:10.1103/PhysRevLett.87.221103].

[31] http://tamago.mtk.nao.ac.jp/decigo/index_E.html.

[32] S. Mollerach, D. Harari, and S. Matarrese, CMB polarization from secondary vector and tensor modes, Phys.Rev. D69 (2004) 063002, [arXiv:astro-ph/0310711], [doi:10.1103/PhysRevD.69.063002].

[33] R. Saito and J. Yokoyama, Gravitational-Wave Constraints on the Abundance of Primordial Black Holes, Prog.Theor.Phys. 123 (2010) 867-886, [arXiv:0912.5317], [doi:10.1143/PTP.123.867]. * Brief entry *.

[34] E. Bugaev and P. Klimai, Bound on induced gravitational wave background from primordial black holes, JETP Lett. 91 (2010) 1-5, [arXiv:0911.0611], [doi:10.1134/S0021364010010017]. * 
Brief entry*.

[35] E. Bugaev and P. Klimai, Constraints on the induced gravitational wave background from primordial black holes, Phys.Rev. D83 (2011) 083521, [arXiv:1012.4697], [doi:10.1103/PhysRevD.83.083521].

[36] E. Bugaev and P. Klimai, Induced gravitational wave background and primordial black holes, Phys.Rev. D81 (2010) 023517, [arXiv:0908.0664], [doi:10.1103/PhysRevD.81.023517].

[37] J. S. Bullock and J. R. Primack, NonGaussian fluctuations and primordial black holes from inflation, Phys.Rev. D55 (1997) 7423-7439, [arXiv:astro-ph/9611106], [doi:10.1103/PhysRevD.55.7423].

[38] P. Ivanov, Nonlinear metric perturbations and production of primordial black holes, Phys.Rev. D57 (1998) 7145-7154, [arXiv:astro-ph/9708224], [doi:10.1103/PhysRevD.57.7145].

[39] P. Pina Avelino, Primordial black hole constraints on non-gaussian inflation models, Phys.Rev. D72 (2005) 124004, [arXiv:astro-ph/0510052], [doi:10.1103/PhysRevD.72.124004].

[40] S. Chongchitnan and G. Efstathiou, Accuracy of slow-roll formulae for inflationary perturbations: implications for primordial black hole formation, JCAP 0701 (2007) 011, [arXiv:astro-ph/0611818], [doi:10.1088/1475-7516/2007/01/011].

[41] J. Hidalgo, The effect of non-Gaussian curvature perturbations on the formation of primordial black holes, arXiv:0708.3875.

[42] E. Bugaev and P. Klimai, Formation of primordial black holes from non-Gaussian perturbations produced in a waterfall transition, arXiv:1112.5601. v2: 11 pages, 4 figures. Several comments and references added. Version accepted by Phys. Rev. D.

[43] J. Chluba, A. L. Erickcek, and I. Ben-Dayan, Probing the inflaton: Small-scale power spectrum constraints from measurements of the CMB energy spectrum, arXiv:1203.2681.

[44] D. H. Lyth and A. R. Liddle, The primordial density perturbation: Cosmology, inflation and the origin of structure, .

[45] M. Drees and E. Erfani, Running Spectral Index and Formation of Primordial Black Hole in Single Field Inflation Models, arXiv:1110.6052.

[46] R. Allahverdi, A. Kusenko, and A. Mazumdar, A-term inflation and the smallness of neutrino masses, JCAP 0707 (2007) 018, [arXiv:hep-ph/0608138], [doi:10.1088/1475-7516/2007/07/018].

[47] C.-M. Lin and K. Cheung, Super Hilltop Inflation, JCAP 0903 (2009) 012, [arXiv:0812.2731], [doi:10.1088/1475-7516/2009/03/012].

[48] C.-M. Lin and K. Cheung, Reducing the Spectral Index in Supernatural Inflation, Phys.Rev. D79 (2009) 083509, [arXiv:0901.3280], [doi:10.1103/PhysRevD.79.083509].

[49] K. Kohri and C.-M. Lin, Hilltop Supernatural Inflation and Gravitino Problem, JCAP 1011 (2010) 010, [arXiv:1008.3200], [doi:10.1088/1475-7516/2010/11/010].

[50] S. Hotchkiss, A. Mazumdar, and S. Nadathur, Observable gravitational waves from inflation with small field excursions, arXiv:1110.5389.

[51] E. D. Stewart, Flattening the inflaton's potential with quantum corrections, Phys.Lett. B391 (1997) 34-38, [arXiv:hep-ph/9606241], [doi:10.1016/S0370-2693(96)01458-X].

[52] L. Covi, D. H. Lyth, and L. Roszkowski, Observational constraints on an inflation model with a running mass, Phys. Rev. D60 (1999) 023509, [arXiv:hep-ph/9809310], [doi:10.1103/PhysRevD.60.023509].

[53] L. Covi and D. H. Lyth, Running-mass models of inflation, and their observational constraints, Phys. Rev. D59 (1999) 063515, [arXiv:hep-ph/9809562], [doi:10.1103/PhysRevD.59.063515].

[54] D. H. Lyth and L. Covi, Observational constraints on the spectral index of the cosmological 
curvature perturbation, Phys. Rev. D62 (2000) 103504, [arXiv:astro-ph/0002397], [doi:10.1103/PhysRevD.62.103504].

[55] L. Covi and D. H. Lyth, Global fits for the spectral index of the cosmological curvature perturbation, Mon. Not. Roy. Astron. Soc. 326 (2001) 885, [arXiv:astro-ph/0008165], [doi:10.1046/j.1365-8711.2001.04466.x].

[56] L. Covi, D. H. Lyth, and A. Melchiorri, New constraints on the running-mass inflation model, Phys. Rev. D67 (2003) 043507, [arXiv:hep-ph/0210395], [doi:10.1103/PhysRevD.67.043507].

[57] L. Covi, D. H. Lyth, A. Melchiorri, and C. J. Odman, The running-mass inflation model and WMAP, Phys. Rev. D70 (2004) 123521, [arXiv:astro-ph/0408129], [doi:10.1103/PhysRevD.70.123521].

[58] P. H. Frampton, M. Kawasaki, F. Takahashi, and T. T. Yanagida, Primordial Black Holes as All Dark Matter, JCAP 1004 (2010) 023, [arXiv:1001.2308], [doi:10.1088/1475-7516/2010/04/023].

[59] M. Kawasaki, A. Kusenko, and T. T. Yanagida, Primordial seeds of supermassive black holes, arXiv:1202.3848.

[60] Numerical Recipes, Third Edition. Cambridge University Press, 2007.

[61] http://www.ligo.caltech.edu/ jzweizig/distribution/LSC_Data/.

[62] http://gwcenter.icrr.u-tokyo.ac.jp/en/.

[63] P. Amaro-Seoane, S. Aoudia, S. Babak, P. Binetruy, E. Berti, et al., eLISA: Astrophysics and cosmology in the millihertz regime, arXiv:1201.3621.

[64] F. A. Jenet, G. Hobbs, W. van Straten, R. Manchester, M. Bailes, et al., Upper bounds on the low-frequency stochastic gravitational wave background from pulsar timing observations: Current limits and future prospects, Astrophys.J. 653 (2006) 1571-1576, [arXiv:astro-ph/0609013], [doi:10.1086/508702].

[65] G. Hobbs, D. Miller, R. Manchester, J. Dempsey, J. Chapman, et al., The Parkes Observatory Pulsar Data Archive, arXiv:1105.5746.

[66] D. Yardley, G. Hobbs, F. Jenet, J. Verbiest, Z. Wen, et al., The Sensitivity of the Parkes Pulsar Timing Array to Individual Sources of Gravitational Waves, arXiv:1005.1667.

[67] http://www .atnf .csiro.au/research/pulsar/ppta/index.php?n=Main.PPTA.

[68] L. A. Boyle, P. J. Steinhardt, and N. Turok, Inflationary predictions reconsidered, Phys.Rev.Lett. 96 (2006) 111301, [arXiv:astro-ph/0507455], [doi:10.1103/PhysRevLett.96.111301].

[69] S. Chongchitnan and G. Efstathiou, Prospects for direct detection of primordial gravitational waves, Phys.Rev. D73 (2006) 083511, [arXiv:astro-ph/0602594], [doi:10.1103/PhysRevD.73.083511].

[70] S. Kuroyanagi, T. Chiba, and N. Sugiyama, Prospects for Direct Detection of Inflationary Gravitational Waves by Next Generation Interferometric Detectors, Phys.Rev. D83 (2011) 043514, [arXiv:1010.5246], [doi:10.1103/PhysRevD.83.043514].

[71] M. Joyce, Electroweak Baryogenesis and the Expansion Rate of the Universe, Phys.Rev. D55 (1997) 1875-1878, [arXiv:hep-ph/9606223], [doi:10.1103/PhysRevD.55.1875].

[72] N. Seto and J. Yokoyama, Probing the equation of state of the early universe with a space laser interferometer, J.Phys.Soc.Jap. 72 (2003) 3082-3086, [arXiv:gr-qc/0305096], [doi:10.1143/JPSJ.72.3082].

[73] L. A. Boyle and A. Buonanno, Relating gravitational wave constraints from primordial nucleosynthesis, pulsar timing, laser interferometers, and the CMB: Implications for the early 
Universe, Phys.Rev. D78 (2008) 043531, [arXiv:0708.2279], [doi:10.1103/PhysRevD.78.043531].

[74] J. Hidalgo, L. A. Urena-Lopez, and A. R. Liddle, Unification models with reheating via Primordial Black Holes, Phys.Rev. D85 (2012) 044055, [arXiv:1107.5669], [doi:10.1103/PhysRevD.85.044055]. Updated to match version accepted by PRD.

[75] K. Nakayama, S. Saito, Y. Suwa, and J. Yokoyama, Space laser interferometers can determine the thermal history of the early Universe, Phys.Rev. D77 (2008) 124001, [arXiv:0802.2452], [doi:10.1103/PhysRevD.77.124001].

[76] K. Nakayama, S. Saito, Y. Suwa, and J. Yokoyama, Probing reheating temperature of the universe with gravitational wave background, JCAP 0806 (2008) 020, [arXiv:0804.1827], [doi:10.1088/1475-7516/2008/06/020].

[77] V. Acquaviva, N. Bartolo, S. Matarrese, and A. Riotto, Second order cosmological perturbations from inflation, Nucl.Phys. B667 (2003) 119-148, [arXiv:astro-ph/0209156], [doi:10.1016/S0550-3213(03)00550-9].

[78] D. Baskaran, L. Grishchuk, and A. Polnarev, Imprints of Relic Gravitational Waves in Cosmic Microwave Background Radiation, Phys.Rev. D74 (2006) 083008, [arXiv:gr-qc/0605100], [doi:10.1103/PhysRevD.74.083008].

[79] S. Dodelson, Modern cosmology, . http://home.fnal.gov/ dodelson/book.html.

[80] M. Abramowitz and I. A. Stegun, Handbook of Mathematical Functions with Formulas, Graphs, and Mathematical Tables. Dover, New York, ninth dover printing, tenth gpo printing ed., 1964.

[81] M. Maggiore, Stochastic backgrounds of gravitational waves, arXiv:gr-qc/0008027.

[82] https://dcc.ligo.org/cgi-bin/DocDB/ShowDocument?docid=2974.

[83] http://www.srl.caltech.edu/ shane/sensitivity/.

[84] H. Kudoh and A. Taruya, Probing anisotropies of gravitational-wave backgrounds with a space-based interferometer: Geometric properties of antenna patterns and their angular power, Phys.Rev. D71 (2005) 024025, [arXiv:gr-qc/0411017], [doi:10.1103/PhysRevD.71.024025].

[85] N. J. Cornish, Detecting a stochastic gravitational wave background with the Laser Interferometer Space Antenna, Phys.Rev. D65 (2002) 022004, [arXiv:gr-qc/0106058], [doi:10.1103/PhysRevD.65.022004].

[86] H. Kudoh, A. Taruya, T. Hiramatsu, and Y. Himemoto, Detecting a gravitational-wave background with next-generation space interferometers, Phys.Rev. D73 (2006) 064006, [arXiv:gr-qc/0511145], [doi:10.1103/PhysRevD.73.064006]. 